\title{
Propositionalization-based relational subgroup discovery with RSD
}

\author{
Filip Železný • Nada Lavrač
}

Received: 24 February 2003 / Revised: 1 December 2004 / Accepted: 27 July 2005 /

Published online: 27 January 2006

C Springer Science + Business Media, Inc. 2006

\begin{abstract}
Relational rule learning algorithms are typically designed to construct classification and prediction rules. However, relational rule learning can be adapted also to subgroup discovery. This paper proposes a propositionalization approach to relational subgroup discovery, achieved through appropriately adapting rule learning and first-order feature construction. The proposed approach was successfully applied to standard ILP problems (East-West trains, King-Rook-King chess endgame and mutagenicity prediction) and two real-life problems (analysis of telephone calls and traffic accident analysis).
\end{abstract}

Keywords Relational data mining $\cdot$ Propositionalization $\cdot$ Feature construction $\cdot$ Subgroup discovery

\section{Introduction}

Classical rule learning algorithms are designed to construct classification and prediction rules (Michie et al., 1994; Clark \& Niblett, 1989; Cohen, 1995). The goal of these predictive induction algorithms is to induce classification/prediction models consisting of a set of rules. On the other hand, opposed to model induction, descriptive induction algorithms (De Raedt \& Dehaspe, 1997; Wrobel \& Džeroski, 1995) aim to discover patterns described in the form of individual rules. Descriptive induction algorithms include association rule learners (e.g., APRIORI (Agrawal et al., 1996)), clausal discovery systems (e.g., CLAUDIEN (De Raedt \& Dehaspe, 1997; De Raedt et al., 2001)), and subgroup discovery systems (e.g., MIDOS

Editors: Hendrik Blockeel, David Jensen and Stefan Kramer

\section{F. Železný $(\bowtie)$}

Czech Technical University, Prague, Czech Republic

e-mail: zelezny@fel.cvut.cz

N. Lavrač

Institute Jožef Stefan, Ljubljana, Slovenia, and Nova Gorica Polytechnic, Nova Gorica, Slovenia e-mail: nada.lavrac@ijs.si 
(Wrobel, 1997; Wrobel, 2001), EXPLORA (Kloesgen, 1996) and SubgroupMiner (Kloesgen \& May, 2002)).

This paper investigates relational subgroup discovery. As in the MIDOS relational subgroup discovery system, a subgroup discovery task is defined as follows: Given a population of individuals and a property of individuals we are interested in, find population subgroups that are statistically 'most interesting', e.g., are as large as possible and have the most unusual statistical (distributional) characteristics with respect to the property of interest.

Notice an important aspect of the above definition: there is a predefined property of interest, meaning that a subgroup discovery task aims at characterizing population subgroups of a given target class. This property indicates that standard classification rule learning algorithms could be used for solving the task. However, while the goal of classification rule learning is to generate models (sets of rules), inducing class descriptions in terms of properties occurring in the descriptions of training examples, in contrast, subgroup discovery aims at discovering individual patterns of interest (individual rules describing the target class).

This paper proposes to adapt classification rule learning to relational subgroup discovery, based on principles that employ the following main ingredients: propositionalization through first-order feature construction, feature filtering, incorporation of example weights into the weighted relative accuracy search heuristic, and implementation of the weighted covering algorithm. Most of the above-listed elements conform to the subgroup discovery methodology proposed by Lavrač et al. (2004); for completeness, these elements are described in Section 3. The main contributions of this paper concern the transfer of this methodology to the multi-relational learning setting. The contributions include substantial improvements of the propositionalization step (compared to the propositionalization proposed by Flach and Lachiche (1999) and Lavrač and Flach (2001)) and an effective implementation of relational subgroup discovery algorithm RSD, employing language and evaluation constraints. Further contributions concern the analysis of the RSD subgroup discovery algorithm in the ROC space, and the successful application of RSD to standard ILP problems (East-West trains, King-Rook-King chess endgame and mutagenicity prediction) and two real-life problem domains (analysis of telephone calls and analysis of traffic accidents).

RSD is available at http://labe.felk.cvut.cz/ zelezny/rsd/. This web page gives access to the RSD system, the user's manual, the data sets (Trains, KRK, Mutagenesis, Telecom $)^{1}$ and the related parameter setting declarations, which enable the reproduction of the experimental results of this paper.

The paper is organized as follows. Section 2 specifies the relational subgroup discovery task, illustrating first-order feature construction and results of rule induction on the wellknown East-West challenge learning problem. It also defines criteria for evaluating the results of subgroup discovery algorithms. In Section 3, the background of this work is explained, including pointers to the related work. Sections 4 and 5 present the main ingredients of the RSD subgroup discovery algorithm: propositionalization through efficient first-order feature construction and constraint-based induction of subgroup descriptions, respectively. Section 6 describes the experimental domains. The results of experiments are presented in Sections 7 and 8 . Section 9 concludes by summarizing the results and presenting plans for further work.

${ }^{1}$ The Traffic dataset is not available due to non-disclosure restrictions. 
Table 1 A subgroup description induced in the Trains domain and the list of definitions of features appearing as literals in the conjunctive antecedent of the rule. Definitions of features are described in the Prolog format

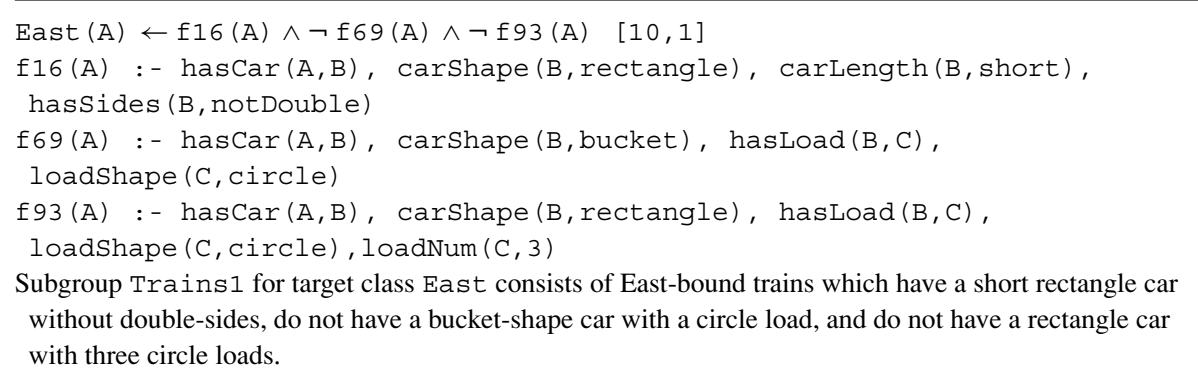

\section{Relational subgroup discovery: Problem definition}

In contrast with predictive induction algorithms which induce models in a rule set form, subgroup discovery aims at finding patterns in the data, described in the form of individual rules. This fact is reflected in the subgroup discovery task definition outlined below.

The input to the RSD relational subgroup discovery algorithm consists of a relational database (possibly deductive, further called the input data) containing (a) one main relation defined by a set of ground facts (training examples), each corresponding to a unique individual and having one argument specifying the class, (b) background knowledge in the form of a Prolog program (possibly including functions and recursive predicate definitions), and (c) syntactic and semantic constraints, defined for the purpose of first-order feature construction and constraint-based subgroup discovery.

The output of RSD is a set of individual rules, each describing a subgroup of individuals whose class distribution differs substantially from the class distribution in the input data set. The rule antecedents (bodies) are conjunctions of symbols of pre-generated first-order features.

The task of relational subgroup discovery is illustrated by a simple East-West trains learning problem, where the subgroup discovery task is to discover patterns in the form of Prolog clauses defining subgroups biased towards one of the two classes: East and West. The original learning task (Michie et al., 1994) was defined as a classification problem and not as a subgroup discovery problem.

Table 1 shows an example subgroup induced from a dataset consisting of 20 trains (10 East-bound and 10 West-bound), where subgroup Trains 1 is described in rule form $H$ $\leftarrow B[T P, F P]$; rule head $H$ denotes the target class, $B$ is the rule body consisting of a conjunction of first-order features, and $T P$ and $F P$ denote the number of true positives (positive examples covered by the rule, correctly predicted as positives) and the number of false positives (negative example covered, incorrectly predicted as positives), respectively. Note that individual feature definitions contain a key variable corresponding to the given individual (the individual 'train'). In evaluation, the truth value of a feature is determined with respect to the individual which instantiates the key variable.

RSD aims at discovering subgroups that are 'most interesting' according to predefined criteria used to measure the interestingness of a subgroup. We consider a subgroup to be interesting if it has a sufficiently large coverage and if it is sufficiently significant (i.e., if it has a sufficiently unusual class distribution compared to the entire training set). In addition, we define quality criteria on sets of subgroups. These are the averages of the coverage and 
significance of the rule set, the rule set complexity and the area under the ROC convex hull formed by the best subgroups. Below we define the quality criteria for individual rules and rule sets.

Coverage. Coverage of a single rule $R_{i}$ is defined as

$$
\operatorname{Cov}\left(R_{i}\right)=\operatorname{Cov}\left(H \leftarrow B_{i}\right)=p\left(B_{i}\right)=\frac{n\left(B_{i}\right)}{N}
$$

where $N$ is the number of all examples and $n\left(B_{i}\right)$ is the number of examples for which the conditions in body $B_{i}$ hold.

Average rule coverage measures the percentage of examples covered on average by one rule of the induced rule set. It is computed as

$$
\operatorname{COV}=\frac{1}{n_{R}} \sum_{i=1}^{n_{R}} \operatorname{Cov}\left(R_{i}\right)
$$

where $n_{R}$ is the number of induced rules.

Complexity. Average rule complexity is measured by a pair of values $R: F$, where $R$ stands for the average number of rules/subgroups per class, and $F$ stands for the average number of features per rule.

Significance. This quantity measures how significantly different the class distribution in a subgroup is from the prior class distribution in the entire example set. We adopt here the significance measure used in the CN2 algorithm (Clark \& Niblett, 1989), where the significance of rule $R_{i}$ is measured in terms of the likelihood ratio statistic of the rule as follows:

$$
\operatorname{Sig}\left(R_{i}\right)=2 \cdot \sum_{j} n\left(H_{j} \cdot B_{i}\right) \cdot \log \frac{n\left(H_{j} \cdot B_{i}\right)}{n\left(H_{j}\right) \cdot p\left(B_{i}\right)} .
$$

where for each class $H_{j}, n\left(H_{j} \cdot B_{i}\right)$ denotes the number of instances of $H_{j}$ in the set where rule body $B_{i}$ holds, $n\left(H_{j}\right)$ is the number of $H_{j}$ instances, and $p\left(B_{i}\right)$ (i.e., rule coverage computed as $\frac{n\left(B_{i}\right)}{N}$ plays the role of a normalizing factor. Note that although for each generated subgroup description one class is selected as the target class, the significance criterion measures the distributional unusualness unbiased to any particular class; as such, it measures the significance of the rule condition only.

Average rule significance, in a rule set consisting of $n_{R}$ rules, is computed as

$$
S I G=\frac{1}{n_{R}} \sum_{i=1}^{n_{R}} \operatorname{Sig}\left(R_{i}\right)
$$

Area under the ROC curve. Each rule (subgroup) is represented by its true positive rate $(T P r)$ and false positve rate $(F P r)^{2}$ as a point in the ROC space, where the $X$-axis corresponds to $F P r$ and the $Y$-axis to $T P r$. Plotting rules in the ROC space allows us to

\footnotetext{
2 The sensitivity or true positive rate of rule $H \leftarrow B$ is computed as $T P r=\frac{T P}{P o s}=\frac{n(H \cdot B)}{n(H)}$, and $F P r=\frac{F P}{N e g}=$ $\frac{n(\bar{H} \cdot B)}{n(\bar{H})}$ is its false alarm or false positive rate.

Springer
} 
compare the quality of individual rules and select the set of best rules, located on the ROC convex hull. To evaluate a set of induced subgroup descriptions, the area under the ROC convex hull (the $A U C$ value) of a set of best subgroup descriptions is computed. Subgroup evaluation in the ROC space is explained in detail in Section 3.6.

\section{Background and related work}

This section provides pointers to related work and presents the background of the proposed approach to relational subgroup discovery.

\subsection{Related subgroup discovery approaches}

Well-known systems in the field of subgroup discovery are EXPLORA (Kloesgen, 1996), MIDOS (Wrobel, 1997, 2001) and SubgroupMiner (Kloesgen \& May, 2002). EXPLORA treats the learning task as a single relation problem, i.e., all the data are assumed to be available in one table (relation), while MIDOS and SubgroupMiner perform subgroup discovery from multiple relational tables. The most important features of these systems, related to this paper, concern the definition of the learning task and the use of heuristics for subgroup discovery. The distinguishing feature of RSD compared to MIDOS and SubgroupMiner is that the latter two systems assume as input the tabular representation of training data and background relations. On the other hand, RSD input data has the form of ground Prolog facts and background knowledge is either in the form of facts or intensional rules, including functions and recursive predicate definitions.

Exception rule learning (Suzuki, 2004) also deals with finding interesting population subgroups. Recent approaches to subgroup discovery, SD (Gamberger and Lavrač, 2002) and CN2-SD (Lavrač et al., 2004), aim at overcoming the problem of inappropriate bias of the standard covering algorithm. Like the RSD algorithm, they use a weighted covering algorithm and modify the search heuristic by example weights. SD and CN2-SD are propositional, while RSD is a relational subgroup discovery algorithm. The subgroup discovery component of RSD shares common basic principles with CN2-SD: the fundamental search strategy and the heuristic function employed therein (the weighted relative accuracy heuristic function is just slightly modified). RSD's subgroup discovery component however implements additional features, such as constraint-based pruning (by detecting when the heuristic function cannot be improved via refinement of the currently explored search node) and various stopping criteria for rule search, employing user-specified constraints.

\subsection{Related propositionalization approaches}

Using relational background knowledge in the process of hypothesis construction is a distinctive feature of relational data mining (Džeroski \& Lavrač, 2001) and inductive logic programming (ILP) (Muggleton, 1992; Lavrač \& Džeroski, 1994).

In propositional learning the idea of augmenting an existing set of attributes with new ones is known as constructive induction. The problem of feature construction has been studied extensively (Pagallo \& Haussler, 1990; Cohen \& Singer, 1991; Oliveira \& SangiovanniVincentelli, 1992, Koller \& Sahami, 1996; Geibel \& Wysotzki, 1996). A first-order counterpart of constructive induction is predicate invention (see e.g., Stahl, 1996 for an overview of predicate invention in ILP). 
Propositionalization (Lavrač \& Džeroski, 1994; Kramer et al., 2001) is a special case of predicate invention enabling the representation change from a relational representation to a propositional one. It involves the construction of features from relational background knowledge and structural properties of individuals. The features have the form of Prolog queries, consisting of structural predicates, which refer to parts (substructures) of individuals and introduce new existential variables, and of utility predicates as in LINUS (Lavrač \& Džeroski, 1994), called properties in Flach and Lachiche (1999), that 'consume' all the variables by assigning properties to individuals or their parts, represented by variables introduced so far. Utility predicates do not introduce new variables. As shown in Section 7.2, the RSD feature construction approach, described in Section 4, effectively upgrades the propositionalization through first-order feature construction proposed by Flach and Lachiche (1999) and Lavrač and Flach (2001).

Related approaches include feature construction in RL-ICET (Turney, 1996), stochastic predicate invention (Kramer et al., 1998) and predicate invention achieved by using a variety of predictive learning techniques to learn background knowledge predicate definitions (Srinivasan \& King, 1996). Earlier approaches, that are closely related to our propositionalization approach, are those used in LINUS (Lavrač \& Džeroski, 1994), and those reported by Zucker and Ganascia (1996, 1998) and Sebag and Rouveirol (1997).

The RSD approach to first-order feature construction can be applied in the so-called individual-centered domains (Flach \& Lachiche, 1999; Lavrač \& Flach, 2001; Kramer et al., 2001), where there is a clear notion of individual, and learning occurs at the level of individuals only. For example, individual-centered domains include classification problems in molecular biology where the individuals are molecules. A simple individual-centered domain is the East-West challenge in Section 2, where trains are individuals.

Individual-centered representations have the advantage of a strong language bias, because local variables in the bodies of rules either refer to the individual or to its parts. However, not all domains are amenable to the approach presented in this paper. In particular, even if in RSD we can use recursion in background knowledge predicate definitions, we cannot induce recursive clauses, and we cannot deal with domains in which there is no clear notion of individuals (e.g., the approach can not be used to learn family relationships and to deal with program synthesis problems).

\subsection{Rule induction using the weighted relative accuracy heuristic}

Rule learning typically involves two main procedures: the search procedure that performs search to find a single rule (described in this section) and the control procedure (the covering algorithm) that repeatedly executes the search in order to induce a set of rules (described in Sections 3.4 and 3.5).

Let us consider a standard propositional rule learner CN2 (Clark \& Niblett, 1987; Clark $\&$ Niblett, 1989). Its search procedure used in learning a single rule performs beam search using classification accuracy of a rule as a heuristic function. The accuracy ${ }^{3}$ of an induced rule of the form $H \leftarrow B$ (where $H$ is the rule head-the target class, and $B$ is the rule body formed of a conjunction of attribute value features) is equal to the conditional probability of head $H$, given that body $B$ is satisfied: $p(H \mid B)$.

\footnotetext{
${ }^{3}$ In some contexts, this quantity is called precision.
} 
The accuracy heuristic $A c c(H \leftarrow B)=p(H \mid B)$ can be replaced by the weighted relative accuracy heuristic. Weighted relative accuracy is a reformulation of one of the heuristics used in MIDOS (Wrobel, 1997) aimed at balancing the size of a group with its distributional unusualness (Kloesgen, 1996).

The weighted relative accuracy heuristic is defined as follows:

$W R A c c(H \leftarrow B)=p(B) \cdot(p(H \mid B)-p(H))$.

Weighted relative accuracy consists of two components: generality $p(B)$, and relative accuracy $p(H \mid B)-p(H)$. The second term, relative accuracy, is the accuracy gain relative to fixed rule $H \leftarrow$ true. The latter rule predicts all instances to satisfy $H$; a rule is only interesting if it improves upon this 'default' accuracy. Another way of viewing relative accuracy is that it measures the utility of connecting rule body $B$ with rule head $H$. Note that it is easy to obtain high relative accuracy with very specific rules, i.e., rules with low generality $p(B)$. To this end, generality is used as a 'weight' which trades off generality of the rule (rule coverage $p(B))$ and relative accuracy $(p(H \mid B)-p(H))$.

In the computation of $A c c$ and WRAcc all probabilities are estimated by relative frequen$\operatorname{cies}^{4}$ as follows:

$$
A c c(H \leftarrow B)=p(H \mid B)=\frac{p(H B)}{p(B)}=\frac{n(H B)}{n(B)}
$$

$W R A c c(H \leftarrow B)=\frac{n(B)}{N}\left(\frac{n(H B)}{n(B)}-\frac{n(H)}{N}\right)$

where $N$ is the number of all the examples, $n(B)$ is the number of examples covered by rule $H \leftarrow B, n(H)$ is the number of examples of class $H$, and $n(H B)$ is the number of examples of class $H$ correctly classified by the rule (true positives).

\subsection{Rule set induction using the covering algorithm}

Two different control procedures for inducing a set of rules are used in CN2: one for inducing an ordered list of rules ${ }^{5}$ and the other for the unordered case. This paper considers only the unordered case in which rules are induced separately for each target class in turn.

For a given class in the rule head, the rule with the best value of the heuristic function (e.g., Acc described in the previous section) is constructed. The covering algorithm then invokes a new rule learning iteration on the training set from which all the covered examples

\footnotetext{
${ }^{4}$ Alternatively, the Laplace estimate (Clark \& Boswell, 1991) and the $m$-estimate (Cestnik, 1990; Džeroski et al., 1993) could also be used.

${ }^{5}$ When inducing an ordered list of rules (a decision list (Rivest, 1987)), the heuristic search procedure finds the best rule body for the current set of training examples, assigning the rule head to the most frequent class of the set of examples covered by the rule. Before starting another search iteration, all examples covered by the induced rule are removed from the training set. The control procedure then invokes search for the next best rule. Induced rules are interpreted as a decision list: when classifying a new example, the rules are sequentially tried and the first rule that covers the example is used for prediction.
} 
of the given target class have been removed, while all the negative examples (i.e., examples that belong to other classes) remain in the training set.

\subsection{Weighted covering algorithm}

In the classical covering algorithm, only the first few induced rules may be of interest as subgroup descriptors with sufficient coverage, since subsequently induced rules are induced from biased example subsets, i.e., subsets including only positive examples not covered by previously induced rules. This bias constrains the population of individuals in a way that is unnatural for the subgroup discovery process, which is aimed at discovering interesting properties of subgroups of the entire population. In contrast, subsequent rules induced by the weighted covering algorithms used in recent subgroup discovery systems SD (Gamberger \& Lavrač 2002) and CN2-SD (Lavrač et al., 2004) allow for discovering interesting subgroup properties in the entire population.

The weighted covering algorithm modifies the classical covering algorithm in such a way that covered positive examples are not deleted from the set of examples which is used to construct the next rule. Instead, in each run of the covering loop, the algorithm stores with each example a count that indicates how many times (with how many induced rules) the example has been covered so far.

Initial weights of all positive examples $e_{j}$ equal 1 . In the first iteration of the weighted covering algorithm all target class examples have the same weight, while in the following iterations the contributions of examples are inverse proportional to their coverage by previously constructed rules; weights of covered positive examples thus decrease according to the formula $\frac{1}{i+1}$, where $i$ is the number of constructed rules that cover example $e_{j}$. In this way the target class examples whose weights have not been decreased will have a greater chance to be covered in the following iterations of the weighted covering algorithm. ${ }^{6}$

\subsection{Subgroup evaluation and WRAcc interpretation in the ROC space}

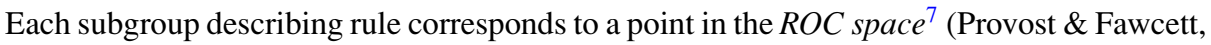
1998), which is used to show classifier performance in terms of false positive rate $F P r$ (the $X$-axis) and true positive rate $\operatorname{TPr}$ (the $Y$-axis). In the ROC space, rules/subgroups whose $T P r / F P r$ tradeoff is close to the diagonal can be discarded as insignificant. Conversely, significant rules/subgroups are those sufficiently distant from the diagonal. The most significant rules define the points in the ROC space from which the ROC convex hull is constructed.

Figure 1 shows examples of ROC diagrams for the Trains domain, plotting the results obtained by the weighted covering and standard covering algorithms, respectively. In this illustrative example we evaluate the induced subgroups on the training set to determine the coordinates of points in the ROC diagram, and not a separate test set, as is normally the case.

\footnotetext{
${ }^{6}$ Whereas this approach is referred to as additive in Lavrač et al. (2004), another option is the multiplicative approach, where for a given parameter $\gamma<1$, weights of positive examples covered by $i$ rules decrease according to $\gamma^{i}$. Both approaches have been implemented in CN2-SD and RSD, but additive weights lead to better results.
}

${ }^{7}$ Abbreviation ROC denotes Receiver Operating Characteristic. 

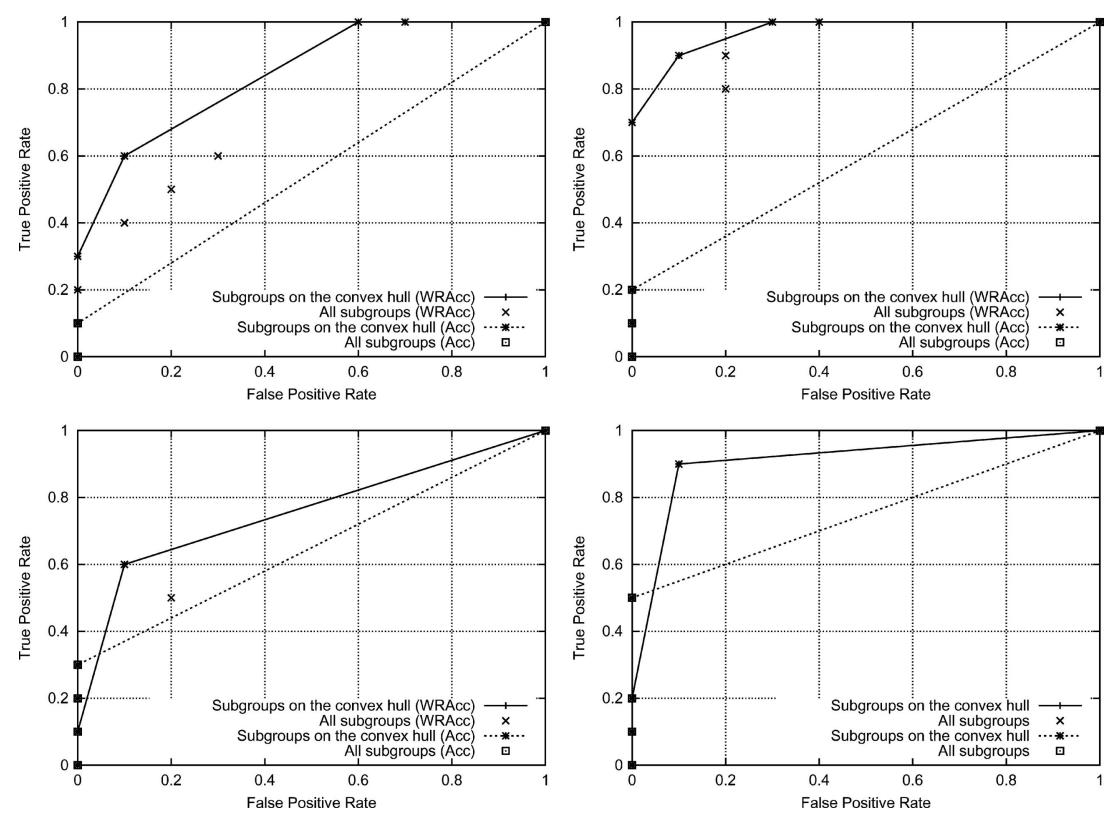

Fig. 1 ROC diagrams for the Trains domain. The left-hand side diagrams show subgroups discovered when West is the target class, and the right-hand side diagrams show subgroups for target class East. The shown subgroups were constructed by the weighted covering algorithm (upper diagrams) and standard covering algorithm (lower diagrams), using the WRAcc and Acc heuristics. Note that subgroups induced using the Acc heuristic are very specific and that they all lie on the $Y$-axis

Weighted relative accuracy of a rule is proportional to the vertical distance of point $(T P r, F P r)$ to the diagonal in the ROC space. To see that this holds, note first that rule accuracy $p(H \mid B)$ is proportional to the angle between the $X$-axis and the line connecting the origin $(0,0)$ with the point $(T P r, F P r)$ depicting the rule in terms of its $T P r / F P r$ tradeoff in ROC space. So, for instance, all points on the $X$-axis have rule accuracy equal 0 , all points on the $Y$-axis have rule accuracy equal 1 , and the diagonal represents subgroups with rule accuracy $p(H)$, i.e., the prior probability of the positive class. Consequently, all point on the diagonal represent insignificant subgroups.

Using relative accuracy, $p(H \mid B)-p(H)$, the above values are re-normalized such that all points on the diagonal have relative accuracy 0 , all points on the $Y$-axis have relative accuracy $1-p(H)=p(\bar{H})$ (the prior probability of the negative class), and all points on the $X$-axis have relative accuracy $-p(H)$. Notice that all points on the diagonal also have $W R A c c=0$. In terms of subgroup discovery, the diagonal thus represents all (insignificant) subgroups with the same target class distribution as present in the whole population; only the generality of these 'average' subgroups increases when moving from left to right along the diagonal. ${ }^{8}$

The area under the ROC curve $(A U C)$ can be used as a quality measure for subgroup discovery. To compare individual subgroup descriptions, a rule/subgroup description is plotted

\footnotetext{
8 This interpretation is slightly different in classifier learning, where the diagonal represents random classifiers that can be constructed without any training.
} 
in the ROC space with its true and false positive rates, and the $A U C$ is calculated. On the other hand, to compare sets of subgroup descriptions induced by different algorithms, we can form the convex hull of the set of points with optimal $T P r / F P r$ tradeoff values. The area under this ROC convex hull indicates the combined quality of the optimal subgroup descriptions, in the sense that it evaluates whether a particular subgroup description has anything to add in the context of all the other subgroup descriptions. This evaluation method has been used in the experiments in this paper. ${ }^{9}$

\subsection{Constraint-based data mining framework for subgroup discovery}

Inductive databases (Imielinsky \& Mannila, 1996) provide a database framework for knowledge discovery in which the definition of a data mining task (Mannila \& Toivonen, 1997) involves the specification of a language of patterns and a set of constraints that a pattern has to satisfy with respect to a given database. In constraint-based data mining (Bayardo, 2002) the constraints that a pattern has to satisfy consist of language constraints and evaluation constraints. The first concern the pattern itself, while the second concern the validity of the pattern with respect to a database. The use of constraints enables more efficient induction as well as focussing the search for patterns on patterns likely to be of interest to the user.

While many different types of patterns have been considered in data mining, constraints have been mostly considered in mining frequent itemsets and association rules, as well as some related tasks, such as mining frequent episodes, Datalog queries, molecular fragments, etc. Few approaches exist that use constraints for other types of patterns/models, such as size and accuracy constraints in decision trees (Garofalakis \& Rastogi, 2000), rule induction with constraints in relational domains including propositionalization (Aronis \& Provost, 1994; Aronis et al., 1996), and using rule sets to maximize the ROC performance (Fawcett, 2001).

In RSD, we use a constraint-based framework to handle the curse of dimensionality present in both procedural phases of RSD: first-order feature construction and subgroup discovery. We apply language constraints to define the language of possible subgroup descriptions, and apply evaluation constraints during rule induction to select the (most) interesting rules/subgroups. RSD makes heavy use of both syntactic and semantic constraints exploited by search space pruning mechanisms. On the one hand, some of the constraints (such as feature undecomposability) are deliberately enforced by the system and pruning based on these constraints is guaranteed not to cause the omission of any solution. On the other hand, additional constraints (e.g., maximum variable depth) may be tuned by the user. These constraints are designed with the intention to most naturally reflect possible user's heuristic expectations or minimum requirements on quantitative evaluations of search results. The combination of the above mentioned strategies controlled by constraints is an original approach to relational subgroup discovery.

\section{RSD propositionalization}

In RSD, propositionalization is performed in three steps:

\footnotetext{
9 This method does not take account of any overlap between subgroups, and subgroups not on the convex hull are simply ignored. An alternative method, employing the combined probabilistic classifications of all subgroups (Lavrač et al., 2004), is beyond the scope of this paper.
} 
- Identifying all expressions that by definition form a first-order feature (Flach \& Lachiche, 1999) and at the same time comply to user-defined mode-language constraints. Such features do not contain any constants and the task can be completed independently of the input data.

- Employing constants. Certain features are copied several times with some variables grounded to constants detected by inspecting the input data. This step includes a simplified version of a feature filtering method proposed in Lavrač et al. (1999).

- Generating propositionalized representation of the input data using the generated feature set, i.e., a relational table consisting of truth values of first-order features, computed for each individual.

\subsection{First-order feature construction}

RSD accepts feature language declarations similar to those used in Progol (Muggleton, 1995). A declaration lists the predicates that can appear in a feature, and to each argument of a predicate a type and a mode are assigned. In a correct feature, if two arguments have different types, they may not hold the same variable. A mode is either input or output; every variable in an input argument of a literal must appear in an output argument of some preceding literal in the same feature. (Flach \& Lachiche, 1999) further dictate the opposite constraint: every output variable of a literal must appear as an input variable of some subsequent literal. Furthermore, the maximum length of a feature (number of contained literals) is declared, along with optional constraints such as the maximum variable depth (Muggleton, 1995), maximum number of occurrences of a given predicate symbol in a feature, etc.

RSD generates an exhaustive set of features satisfying the language declarations as well as the connectivity requirement, which stipulates that no feature may be decomposable into a conjunction of two or more features. For example, the following expression does not form an admissible feature

hasCar(A, B), hasCar(A, C), long(B), long(C)

since it can be decomposed into two separate features. We do not construct such decomposable expressions, as these are redundant for the purpose of subsequent search for rules with conjunctive antecedents. Furthermore, as we will show in the experimental part of the paper, the concept of undecomposability allows for powerful search space pruning. Notice also that the expression above may be extended into an admissible undecomposable feature if a binary property predicate is added:

hasCar(A, B), hasCar(B, C), long(B), long(C), notSame(B, C)

The construction of features is implemented as depth-first, general-to-specific search where refinement corresponds to adding a literal to the currently examined expression. During the search, each search node found to be a correct feature is listed in the output.

Let us determine the crucial complexity factors in the search for features. Let $M_{i}$ be the maximum number of input arguments found in any declared predicate and $M_{o}$ be the analogous maximum for output arguments. A currently explored expression $f$ with literals $l_{1}$, $l_{2}, \ldots, l_{n}, n<L$ (where $L$ is the prescribed maximum feature size) can be refined by adding a literal $l_{n+1}$, which can be one of $D$ different declared predicates. Each input argument of $l_{n+1}$ can hold one of the variables occurring as output variables in $f$ or the key variable linking to an 

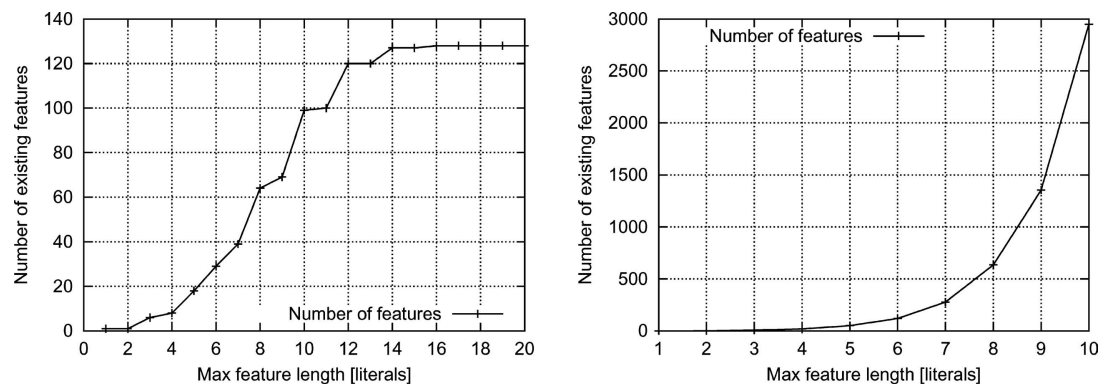

Fig. 2 Left: The number of features as a function of the maximum allowed feature length in the Trains domain where all declared predicates have at most one input argument. Right: The same dependence plotted for the same declaration extended by binary-input property predicate not Same $/ 2$

individual (such as $\mathrm{A}$ in the examples above). There is at most $1+n \cdot M_{o}$ such variables and $l_{n+1}$ has at most $M_{i}$ input arguments. Therefore for each predicate chosen for $l_{n+1}$, there is at most $\left(1+n \cdot M_{o}\right)^{M_{i}}$ choices of argument variables (output arguments acquire new distinct variables), that is, the literal $l_{n+1}$ can be chosen in at most $D \cdot\left(1+n \cdot M_{o}\right)^{M_{i}}$ different ways. The search space thus contains at most $\Pi_{n=1}^{L} D \cdot\left(1+n \cdot M_{o}\right)^{M_{i}} \leq D^{L} \cdot\left(1+L \cdot M_{o}\right)^{M_{i} \cdot L}$ search nodes. Two exponential factors are present in this worst-case estimate: $M_{i}-$ the maximum input arity, and $L-$ the maximum feature length. Out of the two, the former is of less interest to us, since it is typically set to a small constant in common application domains. For example, in the empirical evaluation (Krogel et al., 2003) conducted on six benchmark problems, $M_{\mathrm{i}}$ had the value 1 in four domains and 2 in two domains, in all cases leading to a useful feature set (with respect to the predictive accuracy of the subsequently induced model of data built on the provided features). The latter parameter $L$ is thus a crucial complexity factor of the algorithm-therefore it is used as the independent variable in most of the performance diagrams shown in this paper.

The above worst-case estimate ignores the moding and typing constraints. They may however significantly improve upon the estimate, which we illustrate empirically. The left panel of Figure 2 shows the actual number of features as a function of the maximum allowed definition length in the Trains domain where $M_{i}=1$ (no predicates with more than one input argument are declared). Despite the estimated exponential-in- $L$ growth, the function actually becomes constant at $L=16$. This is no longer the case when binary-input predicate not Same $/ 2$ is further declared (allowing to construct features such as (6) above). Here $M_{i}$ $=2$ and the number of features grows exponentially with $L$.

RSD implements several pruning techniques to reduce the number of examined expressions, while preserving the exhaustiveness of the resulting feature set.

First, suppose that the currently explored expression $f$ of length $n$ contains $o$ output variables not appearing also as input variables in $f$. Let the maximum number of input arguments of a predicate among all available background predicates be $M_{i}$ and $L$ be the maximum length of a feature. Then

\section{Rule 1. Once $L-n \leq \frac{o}{M_{i}}$, prune all descendants of $f$ reached by adding a structural literal to it.}

In other words, if the inequality holds, the algorithm will no longer consider to add structural predicates when refining $f$. By doing so it would introduce one or more new output variables; a simple calculation yields that there would not be enough literal positions left to make all output variables appear also as inputs. 
The following two pruning rules exploit the constraint of feature undecomposability. The constraint is verified by maintaining a set $\vartheta_{\text {eq }}(f)$ of equivalence classes of non-key variables in each explored expression $f$. Two non-key variables $X, Y$ fall in the same equivalence class iff they are connected, i.e., if they both appear as arguments in one literal of the expression, or there is a non-key variable $Z$ such that $X, Z$ are connected and $Z, Y$ are connected. Expression $f$ is a feature only if $\left|\vartheta_{\text {eq }}(f)\right|=1$. Note that a feature may be reached by refining a decomposable node. The following two pruning rules cut off the search subspaces which surely contain only decomposable nodes. Let us call a literal primary if all its input arguments hold the key variable.

Rule 2. If expression $f$ is a non-empty conjunction, prune all its descendants reached by adding a primary property literal to it.

Rule 2 expresses the simple insight that adding a primary property literal to a feature definition will yield a decomposable feature.

\section{Rule 3. Let expression $f$ contain a primary literal $l_{\mathrm{p}}$.}

1. If $l_{p}$ is a property literal, prune all descendants of $f$.

2. If $l_{p}$ is a structural literal and $M_{\mathrm{i}}=1$, prune all descendants reached by adding a primary structural literal to $f$.

The first item of pruning Rule 3 again avoids combining a primary property literal with any other literals. Now consider the case when the maximum input arity of available predicates is equal to 1 (the second item of Rule 3). This is natural in frequent cases when each structural predicate serves for addressing a part or a substructure of a single structure and property predicates do not relate two or more objects. For example, the earlier addressed Trains domain is a typical representant of the situation if the declared language excludes the binary predicate not Same/ 2 relating two cars. The second item of Rule 3 then captures the following idea. To reach a contradiction, let us assume that we can in fact arrive to an admissible feature definition that contains two primary structural literals $l_{1}$ and $l_{2}$. Let $o_{i}$ be some output variable of $l_{\mathrm{i}}(i=1,2)$. Since the maximum input arity is $1, o_{1}$ and $o_{2}$ cannot be connected and the feature would be decomposable, that is, inadmissible.

Finally, it can be shown that Rules 2 and 3 cut off all decomposable nodes in the search tree when $a_{\max }=1$ and therefore we can skip decomposability checks as Rule 4 dictates.

Rule 4. If $M_{\mathrm{i}}=1$, skip all decomposability checks.

Figure 3 illustrates the impact of the described pruning techniques on the efficiency of the feature construction algorithm.

\subsection{Employing constants and feature filtering}

After constructing a constant-free feature set, RSD proceeds to ground selected variables in the features with constants extracted from the input data in the following manner. The user may declare a special property predicate instantiate/1, just like other property predicates. An occurrence of this predicate in a feature with some variable $V$ as its argument specifies that all occurrences of $V$ in the feature should be eventually substituted with a constant. The instantiate/1 literal may appear several times with different variables 

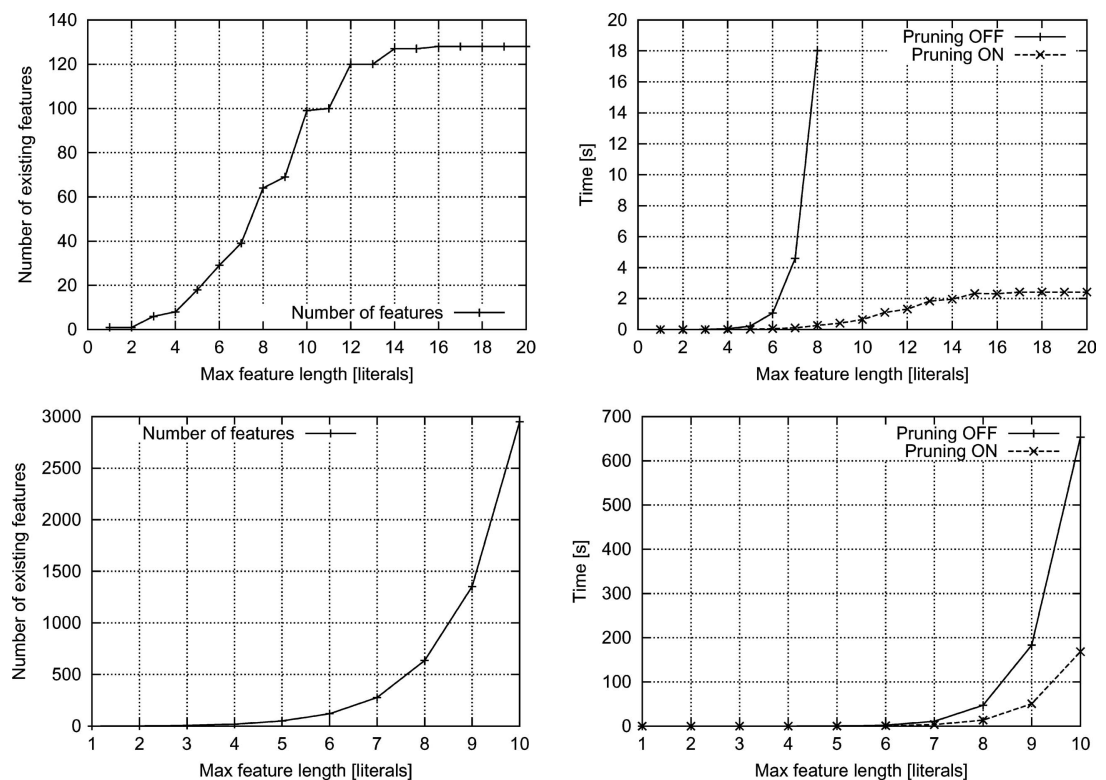

Fig. 3 Empirical example of the impact of search space pruning during syntactic feature construction in the Trains domain. The diagrams on the left show the number of admissible features in the two settings described in Figure 2 (top: at most one input in any declared predicate, bottom: additional binary-input predicate) with the growing maximum feature length: we redisplay these dependencies for ease of comparison. To the right of each of them, we plot the time taken by the algorithm to complete the feature construction in the respective setting with pruning off and on. In the case of only-unary-properties (top), the number of features eventually stops growing, and so does the construction time taken by the pruning-enhanced algorithm as it correctly eliminates the growing search subspaces containing no correct feature. On the contrary, the non-pruning algorithm maintains its exponential time complexity. In the case of additional binary property not Same/2 (bottom), the time dependencies exhibit a similar shape, however, the efficiency gain of the pruning version is still essential

in a single feature. A number of different features are then generated, each corresponding to a possible grounding of the combination of the indicated variables. We consider only those groundings which make the feature true for at least a pre-specified number of individuals.

For example, after consulting the input data, the constant-free expression

hasCar(A, B), hasLoad(B, C), hasShape(C, D), instantiate(D)

is replaced by a set of features, in each of which the instantiate/1 literal is removed and the $\mathrm{D}$ variable is substituted by a constant making the conjunction true for at least one individual, for example

hasCar(A, B), hasLoad(B, C), hasShape(C, rectangle)

Feature filtering takes place within the described step of employing constants and conforms to the following constraints. (a) No feature should have the same Boolean value for all the examples. (b) No two features should have the same Boolean values for all the examples (in this case, a single feature is chosen to represent the class of semantically equivalent 笔 Springer 

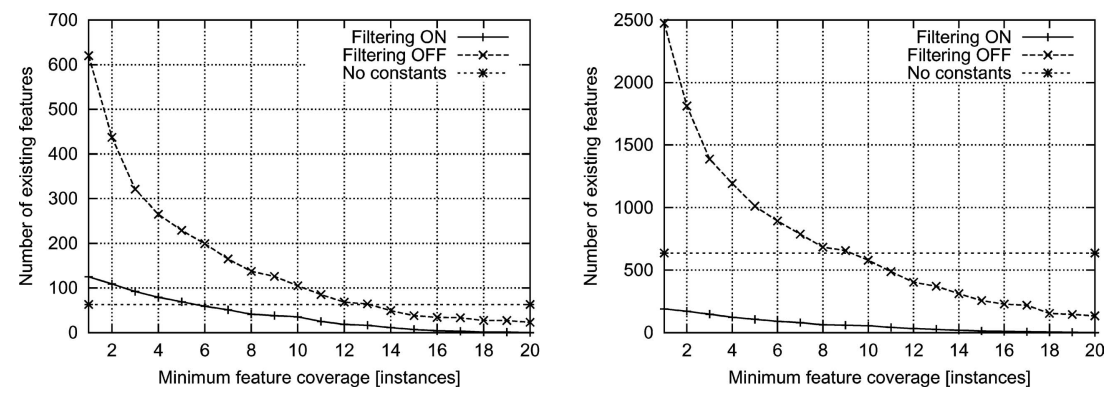

Fig. 4 The impact of feature filtering in the Trains domain in the two respective settings described in Figure 2 (left: at most one input in any declared predicate, right: binary-input predicate not Same/2 added). "No constants": the number of features generated before considering constants. "Filtering off": the number of features after grounding them in several possible ways, each required to make the feature true for at least the number of instances on the X axis. "Filtering on": the same dependency, but discarding a subset of features so that all resulting features have distinct and non-complete coverage

features). (c) A minimum number of examples for which a feature has to be true can be prescribed.

Figure 4 shows an empirical example of the impact of employing constants and feature filtering on the resulting number of features.

\subsection{Generating a propositional representation}

Having constructed the appropriate features, the user can obtain various forms of attributevalue representations of the relational input data. The system can produce either a generic ASCII file of an easily parameterizable format, or files serving as inputs to specific propositional learners. Currently, the supported export formats are those of the RSD subgroup discovery component, the WEKA system (Witten et. al., 1999), the CN2 program (Clark \& Niblett, 1989) and the CN2-SD (Lavrač et al., 2004) propositional subgroup discovery algorithm which uses the same format as CN2.

\section{RSD subgroup discovery}

To identify a set of interesting subgroups, RSD applies the weighted covering algorithm (Section 3.5, while individual rules are found by top-down heuristic beam-search, guided by a variant of the WRAcc search heuristic. The combination of the two principles implies the use of the following modified WRAcc heuristic:

$W R A c c(H \leftarrow B)=\frac{n^{\prime}(B)}{N^{\prime}}\left(\frac{n^{\prime}(H B)}{n^{\prime}(B)}-\frac{n(H)}{N}\right)$

where $N$ is the number of examples, $N^{\prime}$ is the sum of the weights of all examples, $n(H)$ is the number of examples of class $H, n^{\prime}(B)$ is the sum of the weights of all covered examples, and $n^{\prime}(H B)$ is the sum of the weights of all correctly covered examples. Compared to the definition of the WRAcc heuristic used in CN2-SD (Lavrač et al., 2004), the definition of modified WRAcc in Eq. (9) has been improved: in contrast with the $\frac{n^{\prime}(H)}{N^{\prime}}$ computation of 
the prior probability in CN2-SD which changes with changed example weights, in RSD the prior probability computation $\frac{n(H)}{N}$ remains unchanged.

To add a rule to the generated rule set, the rule with the maximum modified WRAcc value is chosen from the set of searched rules not yet present in the rule set generated so far.

The constraints employed in the subgroup search include the language constraint of the maximal number of features considered in the subgroup description as well as several evaluation constraints. These include the minimal value of the significance formula (Clark $\&$ Niblett, 1989) for each subgroup, as well as a minimal value threshold for the modified WRAcc function, which is exploited for sake of pruning.

Two pruning rules are used in the beam-search. According to the first, all refinements of a node will be pruned, if that node stands for a rule covering only instances of the target class, as such a rule cannot be improved by a refinement. Furthermore, if minimal value $t$ is prescribed for $W R A c c$, we prune all refinements of rule $H \leftarrow B$ if the inequality

$\frac{n^{\prime}(B)}{N^{\prime}}\left(1-\frac{n(H)}{N}\right)<t$

holds, as clearly no refinement thereof can yield a WRAcc value higher than the left-hand side of the inequality (weighted coverage will not grow when specializing, while weighted accuracy cannot exceed 1). Constraint (10) thus ensures that no rule is induced whose coverage $\frac{n^{\prime}(B)}{N^{\prime}}$ is so small that even if the rule had perfect accuracy $p(H \mid B)=1$, its coverage vs. relative accuracy tradeoff computed by WRAcc would have been below $t$.

\section{Experimental domains}

In addition to three popular ILP data sets, the East-West trains (Trains), the King-RookKing illegal chess endgame positions (KRK) and mutagenicity prediction (Mutagenesis), we have performed the experimental evaluation of RSD also on two other domains: a real-life telecommunications problem (Telecom) and the analysis of traffic accidents (Traffic). The Trains problem was used in earlier sections for explanatory purposes only.

\subsection{Three ILP domains}

We performed experiments on three popular ILP data sets: Trains, KRK and Mutagenesis.

Trains. We chose the 20 trains East-West challenge (Michie et al., 1994) as an illustrating example earlier in this paper. For these trains, information is given about their cars and the loads of these cars. The original classification task was to discover (low-complexity) models that classify trains as heading East or West. The subgroup discovery task addressed in this paper is to discover subgroups that are sufficiently large and biased towards one of the two classes: East and West.

KRK. In the chess endgame domain White King and Rook versus Black King, taken from (Quinlan, 1990) (first described in Muggleton et al. (1989)), the target relation illegal ( A, B, C, D, E, F) states whether a position where the White King is at file and rank $(A, B)$, the White Rook at $(C, D)$ and the Black King at $(E, F)$ is an illegal White-to-move position. For example, illegal $(\mathrm{g}, 6, \mathrm{C}, 7, \mathrm{C}, 8)$ is a positive example, i.e., an illegal position. Two background predicates are available: $1 t / 2$ expressing the less than relation on a pair of ranks/files, and adj / 2 denoting the adjacency 
relation on such pairs. The data set consists of 1000 instances. The original predictive KRK task aimed at best distinguishing between illegal and legal chess endgame positions, whereas the subgroup discovery task aims at detecting groups of chessboard positions, distinguishable by means of the background relations, which contain an unusually large proportion of illegal/legal positions. In the KRK domain we do not expand features by instantiating variables to constants (as described in Section 4.2). This problem is thus 'purely relational'.

Mutagenesis. The Mutagenesis problem defined in Srinivasan et al. (1996) is a variant of the original data named NS+S2 (also known as B4) that contains information about drugs: their chemical properties, the drugs' atoms and the bonds between the atoms. The original Mutagenesis learning task was to predict whether a drug is mutagenic or not. The separation of data into 'regression-friendly' (188 instances) and 'regression-unfriendly' (42 instances) subsets as described by Srinivasan et al. (1996) is followed in our experiments. Our experiments concentrate on subgroup discovery from the 'regression-friendly' subset consisting of 188 instances.

\subsection{The Telecom domain}

We have applied RSD to a real-life problem in telecommunications. An extensive description of the nature of the data as well as tasks and problems that appear in this domain can be found in Železný et al. (2000, 2002).

The data represent incoming calls (1995 items thereof) to an enterprise. Each call is answered by a human operator and in the usual case further transferred to an attendant distinguished by his/her line number. Further call transfers may also occur. Each sequence of such transfers is tracked by a computerized exchange and related data are stored in a log file. By a suitable transformation thereof, one can form a relation incoming/5, represented by ground facts of the form incoming (date, time, caller, operator, result). The argument result either takes a constant value or is a recursively defined function, so that result $\in\{\mathrm{talk}$, unavailable, transfer $\left(\left[\ln _{1}, \ln _{2}, \ldots, l_{n}\right]\right.$, result $\left.)\right\}$, where $\ln _{1} \ldots \ln _{n}$ denote line numbers to which transfer attempts were made (in the first $n-1$ cases unsuccessfully and in the $n$-th case with outcome result). For example, the following fact

incoming (date $(10,18)$, time $(13,37,29),[0,6,4,8,2,5,6,8,4,9], 32$, transfer $([16,12], \operatorname{transfer}([26], \operatorname{talk})))$

describes a call from phone number 0648256849 at 13:37:29 on 10/18 received by the operator on line 32 . The operator first tried to transfer the caller to line 16 without success, and then transferred him/her successfully to line 12 . The person on line 12 further redirected the caller to line 26 . After a talk with line 26 , the call was terminated.

We divided all instances of incoming transferred calls into 25 classes determined by the line to which the operator tried to transfer the caller first. The arguments of the training instances thus consist of the first four arguments of incoming/5, and the class label $\ln _{1}$. The goal of subgroup discovery is to find subgroups biased to specific classes (destinations of first call transfer) which may be used to support operators' decision making.

Let us now comment on two of the available background relations. The predicate pre$\mathrm{fix}$ (Number, Prefix) is true whenever the second (output) argument is the prefix (of any length) of the first (input) argument. For instance, regarding the example given above, prefix $([0,6,4,8,2,5,6,8,4,9],[0,6,4])$ is true. This background predicate proved useful in previously published results, since it is able to bind callers from the same area, city, company, office etc. 
Table 2 The meaning and the distribution of class values in the UK Traffic challenge data set

\begin{tabular}{lll}
\hline Code & Meaning of class values & $\begin{array}{l}\text { Distribution \% } \\
\text { Class0 }\end{array}$ \\
\hline Class1 & No skidding, jack-knifing or overturning & 64.26 \\
Class2 & Skidded & 22.07 \\
Class3 & Jkidded and overturned & 7.27 \\
Class4 & Jack-knifed & 0.20 \\
Class5 & Overturned & 0.19 \\
\hline
\end{tabular}

Another background predicate (prev \_attempt / 6) reflects the fact that a line desired by the caller may often be determined by looking at the caller's recent attempts to reach a person, i.e., by inspecting past records (w.r.t. the time-label of the current example) in the incoming/ 5 relation. For example, the goal

prev_attempt (date $(10,18)$, time $(13,37,29),[0,6,4,8,2,5,6,8,4,9]$, Line, When, Result)

will succeed with the result

Line $=10$, When = today, Result = unavailable

provided the caller 0648256849 failed to reach line 10 on 10/18 before 13:37:29. Note that the prev_attempt / 6 may yield multiple outputs for a given instantiation of the input arguments.

\subsection{The UK Traffic Accidents Domain}

The UK Traffic data set includes the records of all the accidents that happened on the roads of Great Britain between years 1979 and 1999 (Flach et al., 2003). It is a relational data set consisting of 3 related data tables: the ACCIDENT data, the VEHICLE data and the CASUALTY data. The ACCIDENT data consists of the records of all accidents that happened in the given time period; VEHICLE data includes data about all the vehicles involved in these accidents; CASUALTY data includes the data about all the casualties involved in the accidents. Consider the following example: 'Two vehicles crashed in a traffic accident and three people were seriously injured in the crash'. In terms of the TRAFFIC data set this is recorded as one record in the ACCIDENT data table, two records in the VEHICLE data table and three records in the CASUALTY data table. Every data table is described by around 20 attributes and consists of more than 5 million records.

The UK Traffic challenge data set is formed of a subset of records of the above data set. The task of the challenge was to generate classification models to predict skidding and overturning. As the class attribute Skidding and Overturning appears in the VEHICLE data table, data tables ACCIDENT and VEHICLE were merged in order to make this a nonrelational learning problem. Furthermore, a randomly sampled subset of 5940 records from this merged data table was selected for learning and another sample of 1585 records was selected for testing. The class attribute Skidding and Overturning has six possible values. The meaning of these values and the distribution of class values in the training and test sets are shown in Table 2.

祭 Springer 
Table 3 Examples of generated features

\begin{tabular}{|c|c|}
\hline KRK & f6 (A) : -king1_rank (A, B), rook_rank $(A, C), \operatorname{adj}(C, B)$. \\
\hline Meaning & First king's rank adjacent to rook's rank. \\
\hline Mutagenesis & $\mathrm{f} 12(\mathrm{~A}):-\operatorname{atm}(\mathrm{A}, \mathrm{B}), \operatorname{atm} \_\operatorname{chr}(B, C), 1$ teq_C $(C, 0.142)$. \\
\hline Meaning & A compound contains an atom with charge less or equal to 0.142 . \\
\hline Mutagenesis & f31 $(A)$ :-benzene $(A, B)$, benzene $(A, C)$, connected $(C, B)$. \\
\hline Meaning & Presence of two connected benzene rings in the compound. \\
\hline Telecom & f99 (A) :-ext_number $(A, B), \operatorname{prefix}(B,[0,4,0,7])$. \\
\hline Meaning & Caller's number starts with 0407. \\
\hline Telecom & $\begin{array}{l}\text { f115 (A) :-call_date }(A, B), \operatorname{call}{ }_{-} t i m e(A, C) \text {, ext_number }(A, D) \\
\text { prev_attempt }(B, C, D,[3,1], \operatorname{today,~unavailable).~}\end{array}$ \\
\hline Meaning & The caller of the current call has today tried to reach line 31 , which was unavailable. \\
\hline
\end{tabular}

While the original task of the challenge was to generate classification models to predict skidding and overturning, in our case, the task is to find subgroup descriptions representing interesting skidding and overturning patterns appearing in the Traffic challenge data set.

\section{RSD propositionalization: Experimental evaluation}

This section presents the experimental results. Experiments with feature construction and filtering are illustrated in three domains: KRK, Telecom and Mutagenesis. An experimental comparison of the RSD propositionalization procedure with the original first-order feature construction procedure (Flach \& Lachiche, 1999; Lavrač \& Flach 2001; Kramer et al., 2001) as implemented in SINUS (Krogel et al., 2003) is performed in KRK, Trains and Mutagenesis.

\subsection{Evaluation of feature construction and filtering}

To generate features for KRK and Telecom, we use the background predicates described in the domains descriptions. For the KRK domain, we also allow to generate features in the form of a negation of a complete literal conjunction.

Figure 5 reflects the quantitative characteristics of RSD syntactic feature construction and the effect of pruning in the three domains, from the viewpoint we took in the Trains domain in Figure 3. The efficiency gain by the earlier described search space pruning techniques is not very significant in the KRK domain, but becomes essential with growing feature length in Telecom and Mutagenesis.

Table 3 shows examples of features generated in the KRK, Mutagenesis and Telecom domains.

\subsection{Experimental comparison with SINUS}

This section compares the RSD propositionalization procedure with the first-order feature construction procedure (Flach \& Lachiche, 1999; Lavrač \& Flach, 2001; Kramer et al., 2001) implemented in SINUS (Krogel et al., 2003).

SINUS was first implemented as an extension to the original LINUS transformational ILP learner (Lavrač \& Džeroski, 1994). LINUS performs the transformation to a propositional representation by considering only possible applications of background predicates on the 

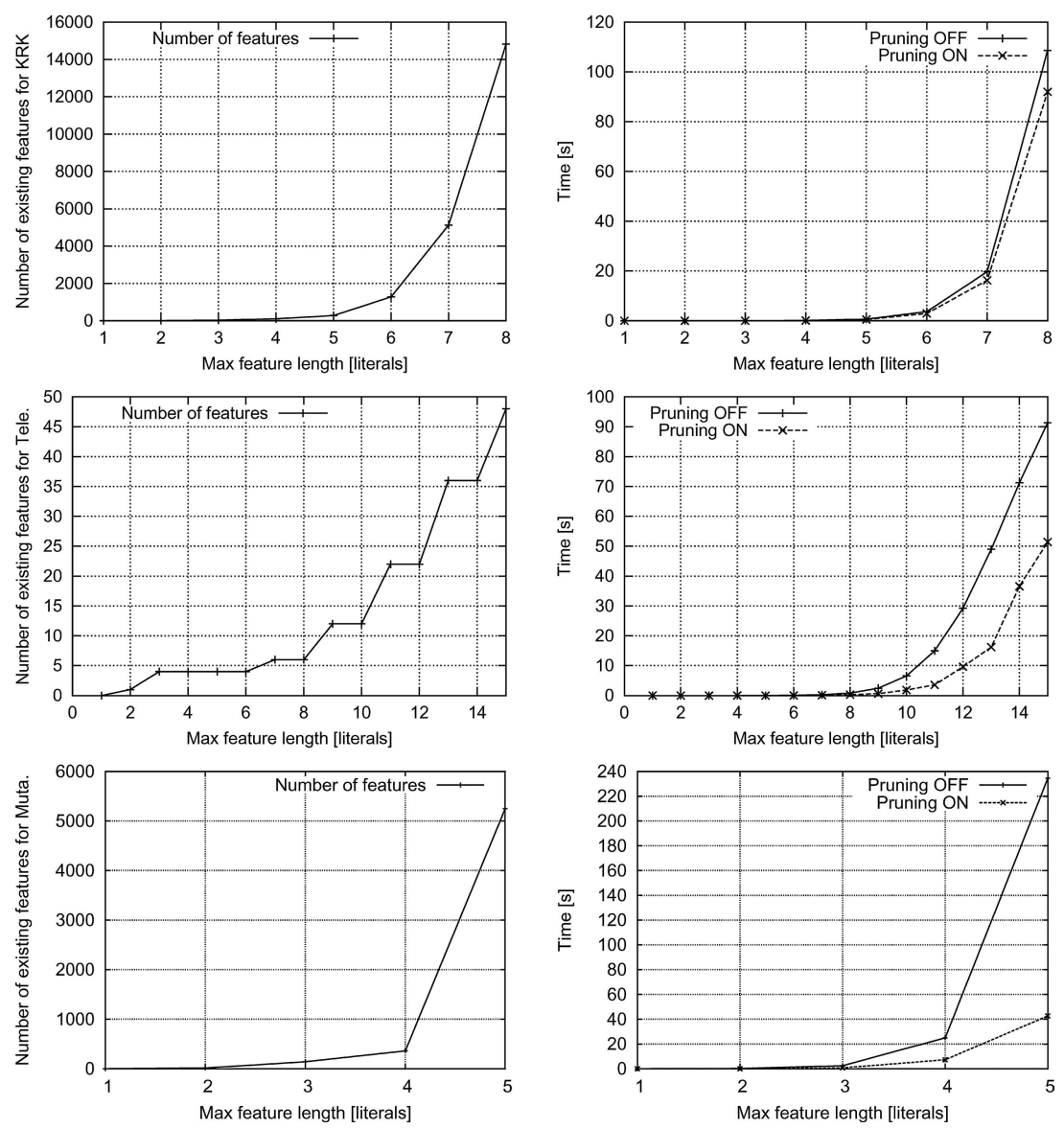

Fig. 5 Number of features (left) and impact of pruning in RSD syntactic feature construction on efficiency (right) in KRK, Telecom and Mutagenesis, shown in dependence to the maximum allowed length of a feature. This viewpoint corresponds to Figure 3 for the example domain of Trains

arguments of the target relation, taking into account the types of arguments. The clauses it learns are constrained. The development of DINUS (Lavrač \& Džeroski, 1994) relaxed this bias so that non-constrained clauses can be constructed provided that the clauses involved are determinate. SINUS was later extended to perform propositionalization by extended first-order feature construction described in Flach and Lachiche (1999), Lavrač and Flach (2001) and Kramer et al., (2001). ${ }^{10}$

The RSD propositionalization has the following advantages compared to SINUS. It provides a declaration language similar to Progol for fine-tuning syntactic constraints on features, it verifies the undecomposability of features and offers improvements (pruning techniques in the feature search, coverage-based feature filtering). These improvements lead to improved efficiency of propositionalization as shown in the experiments of this section.

\footnotetext{
${ }^{10}$ Detailed information about SINUS is available from the SINUS website at http://www.cs.bris.ac.uk/home/rawles/sinus/.
} 
Table 4 Indicators of running times (different platforms, cf. text) and systems providing the feature set for the best-accuracy result in each domain

\begin{tabular}{llllll}
\hline Problem & \multicolumn{2}{c}{ Running times } & & \multicolumn{2}{c}{ J48 Accuracy } \\
\cline { 2 - 3 } \cline { 5 - 6 } & RSD & SINUS & & RSD & SINUS \\
\hline Trains & $<1 \mathrm{sec}$ & 2 to $10 \mathrm{~min}$ & & $65.0 \%$ & $\mathbf{7 0 . 0 \%}$ \\
KRK & $<1 \mathrm{sec}$ & 2 to $6 \mathrm{~min}$ & $\mathbf{9 6 . 3 \%}$ & $95.0 \%$ \\
Mutagenesis & $5 \mathrm{~min}$ & 6 to $15 \mathrm{~min}$ & $\mathbf{9 2 . 5 5 \%}$ & $84.6 \%$ \\
\hline
\end{tabular}

As RSD and SINUS are implemented in different languages (interpreters) and operate on different hardware platforms, exact comparison of runtime efficiency is not possible. As in Krogel et al. (2003), for each domain and system we report the approximate running times, averaged over multiple feature construction runs, varying in the language constraints and producing feature sets of different sizes. RSD ran under the Yap Prolog on a Celeron $800 \mathrm{MHz}$ computer with $256 \mathrm{MB}$ of RAM. SINUS ran under SICStus Prolog ${ }^{11}$ on a Sun Ultra 10 computer. Table 4 shows running times of the propositionalization systems on the learning tasks (with best results indicated in bold). The table also provides 10-fold stratified cross-validation accuracy results of applying the J48 propositional learner (a reimplementation of C4.5 (Quinlan, 1993) available in WEKA (Witten \& Frank, 1999)), supplied with propositionalized data based on feature sets of varying size obtained from the two propositionalization systems. To test the performance of the two systems, producing the accuracy results in Table 4, RSD and SINUS were used to construct features by different parameter settings; the results of 10-fold stratified cross-validation for the best setting are shown.

Different performance of RSD and SINUS are due to their different ways of constraining the language bias. RSD wins in the KRK domain and Mutagenesis. While the gap on KRK seems little significant, the result obtained on Mutagenesis with RSD's 25 features $^{12}$ is competitive to the best results reported in the ILP literature. Whether the apparent efficiency superiority of RSD w.r.t SINUS is due to the RSD's pruning mechanisms, or the implementation in the faster Yap Prolog, or a combined effect thereof has to be investigated in more depth in future work.

\section{RSD subgroup discovery: Experimental evaluation}

For the experimental domains Trains, KRK, Mutagenicity and Telecom, parts of the experimental settings overlap, and parts are unique for each of the domains. The common parts of the experimental procedures are outlined below. A separate evaluation procedure is used in the Traffic challenge domain as the goal is to compare RSD with SubgroupMiner in terms of the $A U C$ performance.

The RSD algorithm was run as follows.

\footnotetext{
${ }^{11}$ It should be noted that SICStus Prolog is generally considered to be several times slower than Yap prolog.

12 The longest have 5 literals in their bodies. Prior to irrelevant-feature filtering conducted by RSD, the feature set had 533 features.
} 
- For each dataset we first ran the RSD propositionalization procedure. We generated a set of features and-when applicable-expanded the set with features containing variable instantiations. We then used the feature sets to produce a propositional representation of each of the data sets. The results of this evaluation were presented in Section 7.1.

- We then ran the RSD subgroup discovery procedure to construct interesting subgroups from the propositional data. In this procedure we have subsequently exchanged the RSD's WRAcc heuristic with the standard accuracy (Acc) heuristic, and the RSD's weighted covering algorithm with the standard covering algorithm, thereby testing four configurations of the method. Results of each are compared in terms of subgroup interestingness criteria. See the list of subgroup evaluation criteria in Section 2 and the results of the evaluation in Section 8.1.

The generation of stratified cross-validation splits and subsequent assessment of resulting rules is done automatically by the RSD system. The experiments are repeatable, as the RSD package available at http : //labe.felk.cvut.cz/ zelezny/rsd contains scripts needed to conduct the experimental procedures automatically to achieve the results presented below.

\subsection{Subgroup discovery evaluation results}

In the experiments we used different variants of the RSD rule learning algorithm by altering the covering strategy and the heuristic function used to construct sets of subgroup-describing rules.

Achieved results and characteristics of discovered rules are shown in Table 5, where Algo refers to the combination of the search heuristic ( $A$ - accuracy, $W$ - weighted relative accuracy) and the covering algorithm $(C$ - covering, $W$ - weighted covering using additive weights). Results are evaluated in terms of the following interestingness criteria: $S I G$ significance, $C O V$ - coverage, $A U C$ - area under the ROC curve, $R: F$ - average number of rules per class : average number of features per rule, $R^{\prime}: F^{\prime}$ - the same as above, but considering only the rules on the ROC convex hull. The reported results are averages obtained in 10-fold stratified cross-validation, along with the corresponding standard deviations.

Rule generation for a given class was terminated if the search space was completely explored or the maximal number of subgroup rules was generated (10 subgroup rules generated for KRK and Telecom, and 5 for Mutagenesis).

The most important observation about the results in Table 5 is that, in terms of all the quality criteria, the RSD's WRAcc heuristic very significantly improves the performance compared to the standard accuracy heuristic. Overall, the combination of the WRAcc heuristic with the strategy of example weighting used in the weighted covering algorithm yields the best results. This agrees with the findings in (Lavrač et al., 2004), where a more extensive empirical evaluation was conducted on a collection of (non-relational) subgroup discovery problems, comparing the $\mathrm{CN} 2$ algorithm with $\mathrm{CN} 2$ incorporating a variant of the WRAcc heuristic, and further with the CN2-SD system (which incorporates a variant of the WRAcc heuristic and the weighted covering algorithm). These three algorithms roughly correspond to the methods we denote above (in Table 5) as $A C, W C$, and $W W$, respectively. The combination of the accuracy heuristic with example weighting $A W$ performs worse in the domains considered.

\subsection{Expert analysis of induced subgroups}

We now present selected subgroups, discovered by RSD in the KRK and Telecom domains, to illustrate the character of induced rules. We also present their pie-chart visualization. 
Table 5 Results of ten-fold cross-validation obtained by the RSD algorithm in the KRK, Mutagenesis and Telecom domains (with standard deviations in parentheses)

\begin{tabular}{|c|c|c|c|c|c|}
\hline \multirow[b]{2}{*}{ Algo } & \multicolumn{3}{|c|}{ Performance } & \multicolumn{2}{|c|}{ Complexity } \\
\hline & $\overline{S I G}$ & $\mathrm{COV}$ & $A U C$ & $R: F$ & $R^{\prime}: F^{\prime}$ \\
\hline \multicolumn{6}{|c|}{ KRK } \\
\hline$A C$ & $\begin{array}{l}2.29 \\
(0.59)\end{array}$ & $\begin{array}{l}1.82 \% \\
(0.37)\end{array}$ & $\begin{array}{l}0.54 \\
(0.01)\end{array}$ & $\begin{array}{l}10.00: 2.19 \\
(0.00: 0.04)\end{array}$ & $\begin{array}{l}10.00: 2.19 \\
(0.00: 0.04)\end{array}$ \\
\hline$A W$ & $\begin{array}{l}2.59 \\
(0.84)\end{array}$ & $\begin{array}{l}1.80 \% \\
(0.48)\end{array}$ & $\begin{array}{l}0.54 \\
(0.01)\end{array}$ & $\begin{array}{l}10.00: 2.57 \\
(0.00: 0.05)\end{array}$ & $\begin{array}{l}10.00: 2.57 \\
(0.00: 0.05)\end{array}$ \\
\hline$W C$ & $\begin{array}{l}9.12 \\
(1.28)\end{array}$ & $\begin{array}{l}7.92 \% \\
(0.44)\end{array}$ & $\begin{array}{l}\mathbf{0 . 7 2} \\
(0.02)\end{array}$ & $\begin{array}{l}\mathbf{7 . 5 0}: \mathbf{2 . 8 0} \\
(0.00: 0.06)\end{array}$ & $\begin{array}{l}6.50: 2.78 \\
(0.44: 0.06)\end{array}$ \\
\hline$W W$ & $\begin{array}{l}\mathbf{1 1 . 1 4} \\
(2.05)\end{array}$ & $\begin{array}{l}\mathbf{1 0 . 6 4 \%} \\
(0.64)\end{array}$ & $\begin{array}{l}\mathbf{0 . 7 2} \\
(0.02)\end{array}$ & $\begin{array}{l}10.00: 2.77 \\
(0.00: 0.04)\end{array}$ & $\begin{array}{l}\mathbf{6 . 0 0}: \mathbf{2 . 6 4} \\
(0.52: 0.06)\end{array}$ \\
\hline \multicolumn{6}{|c|}{ Mutagenesis } \\
\hline$A C$ & $\begin{array}{l}1.99 \\
(0.92)\end{array}$ & $\begin{array}{l}11.33 \% \\
(3.74)\end{array}$ & $\begin{array}{l}0.69 \\
(0.07)\end{array}$ & $\begin{array}{l}10.00: 2.16 \\
(0.00: 0.07)\end{array}$ & $\begin{array}{l}10.00: 2.16 \\
(0.00: 0.07)\end{array}$ \\
\hline$A W$ & $\begin{array}{l}1.33 \\
(1.05)\end{array}$ & $\begin{array}{l}7.62 \% \\
(4.88)\end{array}$ & $\begin{array}{l}0.58 \\
(0.06)\end{array}$ & $\begin{array}{l}10.00: 2.50 \\
(0.00: 0.11)\end{array}$ & $\begin{array}{l}10.00: 2.50 \\
(0.00: 0.11)\end{array}$ \\
\hline$W C$ & $\begin{array}{l}4.22 \\
(1.22)\end{array}$ & $\begin{array}{l}35.81 \% \\
(6.44)\end{array}$ & $\begin{array}{l}0.86 \\
(0.06)\end{array}$ & $\begin{array}{l}\mathbf{3 . 7 0}: \mathbf{1 . 7 3} \\
(0.82: 0.33)\end{array}$ & $\begin{array}{l}\mathbf{2 . 3 0}: \mathbf{1 . 6 2} \\
(0.48: 0.22)\end{array}$ \\
\hline$W W$ & $\begin{array}{l}\mathbf{7 . 4 8} \\
(1.28)\end{array}$ & $\begin{array}{l}\mathbf{4 0 . 5 8 \%} \\
(4.74)\end{array}$ & $\begin{array}{l}\mathbf{0 . 9 0} \\
(0.04)\end{array}$ & $\begin{array}{l}10.00: 2.63 \\
(0.00: 0.07)\end{array}$ & $\begin{array}{l}6.50: 2.43 \\
(0.97: 0.11)\end{array}$ \\
\hline \multicolumn{6}{|c|}{ Telecom } \\
\hline$A C$ & $\begin{array}{l}2.90 \\
(0.38)\end{array}$ & $\begin{array}{l}0.37 \% \\
(0.05)\end{array}$ & $\begin{array}{l}0.55 \\
(0.02)\end{array}$ & $\begin{array}{l}7.36: 2.39 \\
(0.12: 0.04)\end{array}$ & $\begin{array}{l}6.88: 2.47 \\
(0.19: 0.04)\end{array}$ \\
\hline$A W$ & $\begin{array}{l}2.56 \\
(0.52)\end{array}$ & $\begin{array}{l}0.26 \% \\
(0.04)\end{array}$ & $\begin{array}{l}0.55 \\
(0.02)\end{array}$ & $\begin{array}{l}9.96: 2.56 \\
(0.07: 0.03)\end{array}$ & $\begin{array}{l}9.60: 2.61 \\
(0.07: 0.03)\end{array}$ \\
\hline$W C$ & $\begin{array}{l}11.29 \\
(1.71)\end{array}$ & $\begin{array}{l}\mathbf{4 . 9 8 \%} \\
(0.54)\end{array}$ & $\begin{array}{l}0.67 \\
(0.02)\end{array}$ & $\begin{array}{l}\mathbf{6 . 1 2}: \mathbf{2 . 1 7} \\
(0.16: 0.04)\end{array}$ & $\begin{array}{l}\mathbf{5 . 2 0}: \mathbf{2 . 2 8} \\
(0.16: 0.04)\end{array}$ \\
\hline$W W$ & $\begin{array}{l}\mathbf{1 2 . 0 0} \\
(1.05)\end{array}$ & $\begin{array}{l}4.07 \% \\
(0.41)\end{array}$ & $\begin{array}{l}\mathbf{0 . 7 0} \\
(0.01)\end{array}$ & $\begin{array}{l}9.64: 2.06 \\
(0.12: 0.01)\end{array}$ & $\begin{array}{l}6.68: 2.29 \\
(0.20: 0.03)\end{array}$ \\
\hline
\end{tabular}

Table 6 A subgroup description induced in the KRK domain in the form $H \leftarrow B[T P, F P]$, its interpretation and definitions of features appearing as literals in the conjunctive antecedent of a rule describing the subgroup

Subgroup KRK1 for target class: legal

$\operatorname{legal}(\mathrm{A}) \leftarrow \mathrm{f} 139(\mathrm{~A}) \wedge \mathrm{f} 145(\mathrm{~A}) \wedge \mathrm{f} 133(\mathrm{~A})[279,4]$

f139 (A) : - not (rook_rank $(A, B), \operatorname{king} 2 \_r a n k(A, C), \operatorname{adj}(C, C), \operatorname{adj}(C, B)$ ).

f145 (A) : - not (rook_file (A,B), king2_file $(A, C), \operatorname{adj}(C, C), \operatorname{adj}(C, B))$.

f133 (A) : - not (king1_file (A, B), king2_file $(A, C), \operatorname{adj}(C, C), \operatorname{adj}(C, B))$.

Configurations where rook's rank is not adjacent to second king's rank and rook's file not adjacent to second king's file and first king's file not adjacent to second king's file (note that adj ( C, C) is always true, i.e. redundant).

Table 6 presents a subgroup, induced in the KRK domain, and lists the Prolog notation of the features used as antecedent literals in the rule that describes the subgroup. The graphical presentation of the class distribution and coverage of the subgroup, illustrated at the righthand side of Figure 6, is complemented in Table 6 by a verbal description of the subgroup, together with the number of true positive $(T P)$ and false positive $(F P)$ examples covered. 


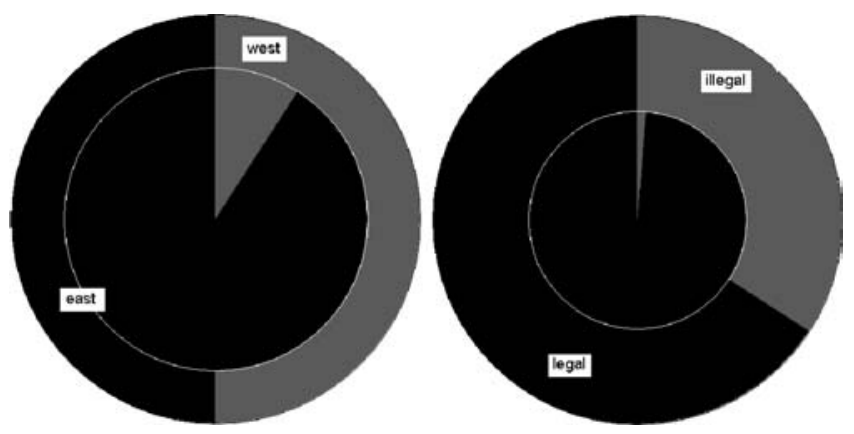

Fig. 6 Left: Subgroup Trains1 described in Table 1 of Section 2. Right: Subgroup KRK1 described in Table 6 of this section

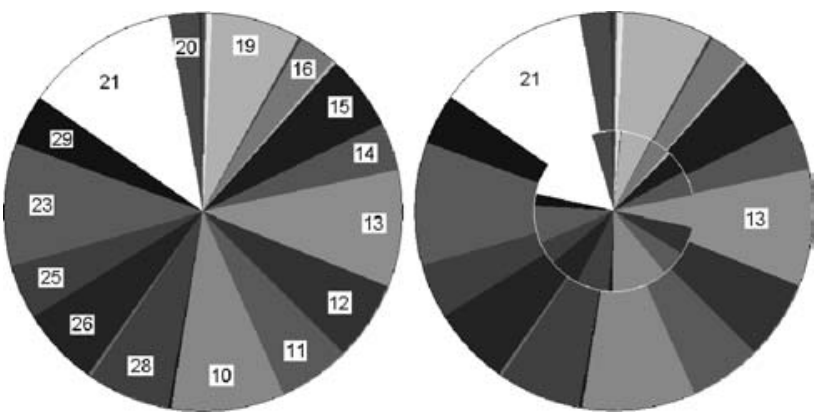

Fig. 7 Left: Prior distribution of classes in the Telecom domain. Right: Subgroup Tele1 described in Table 7 of this section
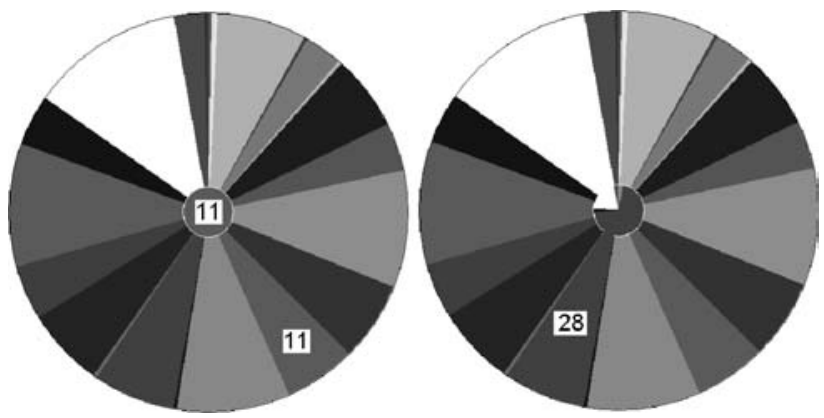

Fig. 8 Left: Subgroup Tele2, Right: Subgroup Tele4, both described in Table 7 of this section

Figures 6-8 show pie-chart representations of the distributional characteristics of induced subgroups. In the outer pie, each area representing a class is proportional to the frequency of that class in the data set. Similarly, areas in the inner pie are proportional to the frequencies of corresponding classes in the subgroup. The ratio of the overall area of the inner pie to the area of the whole pie is the ratio between the number of instances included in the subgroup in and the number of all the instances in the respective data set.

In the Telecom domain, although exactly one class is the target, it often follows from the illustrated posterior distribution that the rule consequent can naturally be interpreted as a Springer 
Table 7 Telecom subgroup descriptions in the form $H \leftarrow B[T P, F P]$, definitions of used features, and subgroup interpretation including expert's comments

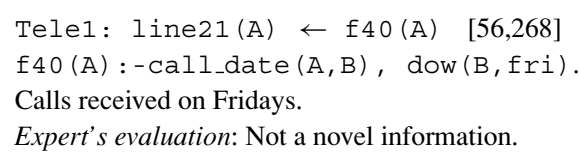

Tele2: line11 $(\mathrm{A}) \leftarrow \mathrm{f} 132(\mathrm{~A}) \quad[32,0]$

f132(A) :-ext_number (A, B), $\operatorname{prefix}(B,[8,5,1,3,1,1,1,1])$.

Calls received from number 85131111 .

Expert's explanation: The caller is the secretary's husband. She does not have a direct-access line, thus this call is transferred by an operator.

Expert's evaluation: Novel information.

Remark. Although the last literal formally identifies a prefix of the calling number, it is in fact the complete number of the caller.

Tele3: line21 $(\mathrm{A}) \leftarrow$ f54 $(\mathrm{A}) \quad[81,254]$

f54 (A) : -ext_number $(A, B), \operatorname{prefix}(B,[0,4])$.

Calls received from a number that starts with 04 .

Expert's explanation: Prefix 04 is too general (code covers a large area) to find an explanation.

Expert's evaluation: Novel information. Uncertain.

Tele4: line28 $(A) \leftarrow$ f7 $(A) \quad[22,11]$

$\mathrm{f} 7(\mathrm{~A})$ :- call_date $(\mathrm{A}, \mathrm{B}), \operatorname{call}$ _time $(\mathrm{A}, \mathrm{C})$,

ext_number (A, D), prev_attempt (B, C, D, [2,1], last_hour, unavailable) .

Calls received from a caller who has in the last hour attempted to directly (not through an operator) reach line 28, which was unavailable.

Expert's explanation: It is plausible that people try line 28 as the second attempt when line 21 is unavailable. Subgroup probably mostly covers people with technical difficulties with a product sold by person on line 21.

Expert's evaluation: Novel information.

disjunction of classes. This applies in cases when a subgroup contains instances of only a few classes, as opposed to the prior distribution of 25 classes. ${ }^{13}$

We now present the descriptions of some of the discovered subgroups in Telecom, with comments from the domain expert on the descriptions in Table 7 and the distributional characteristics of the subgroups in terms of the number of true positives $(T P)$ and false positives $(F P)$.

Expert analysis of the induced rules shows that some of them identify novel and interesting information. Especially revealing are the comments related to the changes of class frequency associated with the rules, as illustrated in the pie-charts. In the overall distribution, calls to line 21 are the most common. The expert commented that this reflects his expectations, as the person at line 21 is a marketer, and people interested in products call this line most frequently. In subgroup Tele1, there is (a) an increase in line 21 frequency: clients not receiving an ordered package often wait until Friday and then complain with line 21; and (b) a decrease in line 13 frequency: the person at line 13 mostly collaborates with dealers who have less business on Fridays. For subgroup Tele4 there is (a) an increase in line 28 frequency: repeated attempts to reach line 28, and (b) an increase in line 21

\footnotetext{
${ }^{13}$ Note that only 15 classes are visible in the outer pies in the Telecom domain, as instances of the remaining 10 classes are rare and thus below the graphics resolution.
} 
Table 8 Performance comparison of Aleph and the AC and WW variants of the RSD algorithm in terms of average significance $(S I G)$, coverage $(C O V)$ and area under the ROC curve $(A U C)$

\begin{tabular}{llrrr}
\hline Domain & Algo & \multicolumn{1}{c}{ SIG } & COV & AUC \\
\hline KRK & Aleph & 2.30 & $1.18 \%$ & 0.56 \\
& AC & 2.29 & $1.82 \%$ & 0.54 \\
& $W W$ & 11.14 & $10.64 \%$ & 0.72 \\
\multirow{3}{*}{ Muta } & Aleph & 1.40 & $10.39 \%$ & 0.72 \\
& AC & 1.99 & $11.33 \%$ & 0.69 \\
& $W W$ & 7.48 & $40.58 \%$ & 0.90 \\
\multirow{3}{*}{ Telecom } & Aleph & 5.24 & $0.27 \%$ & 0.75 \\
& AC & 2.90 & $0.37 \%$ & 0.55 \\
& $W W$ & 12.00 & $4.07 \%$ & 0.70 \\
\hline
\end{tabular}

frequency: the person at line 28 works as technical support for products sold by person on line 21.

\subsection{Experimental comparison with Aleph}

Having compared the four discovery strategy variants within the single RSD system in Section 8.1, we now compare RSD with the relational classification rule learner Aleph, using again the KRK, Telecom and Mutagenesis datasets. Aleph is a system for relational rule induction implementing the basic principles described in Muggleton (1995). Unlike RSD, Aleph directly conducts search in a set of first-order rules without prior propositionalization of the learning data. As a classificatory induction system, Aleph uses the standard (nonweighted) example covering strategy for constructing a rule set, and function $f(R)=n^{+}(R)-$ $n^{-}(R)$ is used as the search heuristic, where $n^{+}(R)$ and $n^{-}(R)$ are the numbers of positive and negative examples covered by rule $R$, respectively.

Table 8 compares the qualities of subgroups corresponding to rules discovered by Aleph with those produced by the $A C$ and $W W$ variants of RSD (the last two are repeated from Table 5). The results were obtained by the same measurement procedure as in Section 8.1. As Aleph is a binary-class learner, the 25 class Telecom problem was split into 25 tasks, each time with one class representing the positive examples and all other classes representing the negative examples. The final results are averaged over all the 25 learning tasks.

The basic trend observed in the results is that subgroups corresponding to rules found by Aleph exhibit qualities much closer to those discovered by the $A C$, rather than the $W W$ variant of RSD. The main difference between Aleph and the $A C$ variant of RSD is the initial propositionalization of data performed by the latter algorithm. The results thus suggest that propositionalizing the learning data does not incur, at least in the tested domains, a detrimental effect on the resulting subgroup qualities.

\subsection{Experimental comparison with SubgroupMiner}

We further compared RSD to the relational subgroup discovery system SubgroupMiner (Kloesgen \& May, 2002). While RSD accepts intensional definitions of background relations, SupgroupMiner requires the input to be in the form of relational tables (ground facts). 
This requirement on data form is met in the Traffic challenge problem, which already served for a mutual comparison of subgroup discovery algorithms in Kavšek and Lavrač (2004).

The task addressed is to find subgroup descriptions representing interesting skidding and overturning patterns appearing in the Traffic challenge data set consisting of 5940 randomly sampled records from the original ACCIDENT and VEHICLE tables. Recall that the class attribute Skidding and Overturning has six possible values: Class0: No skidding, jack-knifing or overturning; Class1: Skidded; Class2: Skidded and overturned; Class3: Jack-knifed; Class4: Jack-knifed and overturned; and Class5: Overturned.

We here adhere to the experimental setting of Kavšek and Lavrač (2004), following the same results presentation and comparison of the ROC convex hulls obtained from best subgroup descriptions induced by each of the two compared subgroup discovery systems.

The $T P r$ and $F P r$ characteristics of subgroup descriptions induced by the two respective systems for three selected values of the target attribute (Class0: No skidding, jackknifing or overturning; Class1: Skidded; and Class5: Overturned) are plotted in Figure 9. In these experiments, RSD was run in the $W W$ setting of Table 5, using the weighted covering algorithm and the modified weighted relative accuracy heuristic. The two systems identify best subgroups on the ROC convex hull of roughly similar quality. However, SubgroupMiner induces a much larger set of subgroups, some lying much below the ROC curve.

\section{Conclusions and further work}

This paper presents an approach to relational subgroup discovery, whose origins are based on the recent developments in subgroup discovery (Wrobel, 2001; Lavrač et al., 2004) and propositionalization through first-order feature construction (Flach \& Lachiche, 1999; Lavrač \& Flach, 2001; Kramer et al., 2001). It presents constraint-based relational subgroup discovery algorithm RSD which transforms a relational subgroup discovery problem to a propositional one, through efficient first-order feature construction. Efficiency is boosted mainly through exploiting language and evaluation constraints for pruning.

Concerning propositionalization, our empirical results demonstrate that the strategy implemented in RSD represents an advance over the original first-order feature construction procedure (Flach \& Lachiche, 1999; Lavrač \& Flach, 2001) in terms of runtime efficiency. Although the efficiency gain is rather negligible in the KRK domain, it is significant in both the Telecom and Mutagenesis domains. Furthermore, additional experiments with classificatory induction using the generated propositional form indicate that RSD produces features leading to high classification accuracy. Lastly, absolute-runtime comparisons with SINUS which implements the propositionalization procedure described in Flach and Lachiche (1999) and Lavrač and Flach (2001) indicate the superiority of RSD, although these figures are not conclusive due to the inherently different software and hardware used by RSD and SINUS.

Following the data propositionalization step, four variants of the core subgroup discovery RSD algorithm were tested, by combining the standard accuracy and the weighted accuracy search heuristic used in the construction of individual rules, with the standard covering and weighted covering algorithm used in the construction of a set of rules. The WRAcc heuristic combined with the weighted covering algorithm is the preferred combination (due to an 
Fig. 9 The traffic challenge: The ROC points corresponding to subgroup descriptions generated by RSD and SubgroupMiner for three classes of the target attribute. For visual clarity, the convex hull is interpolated only for the RSD subgroups

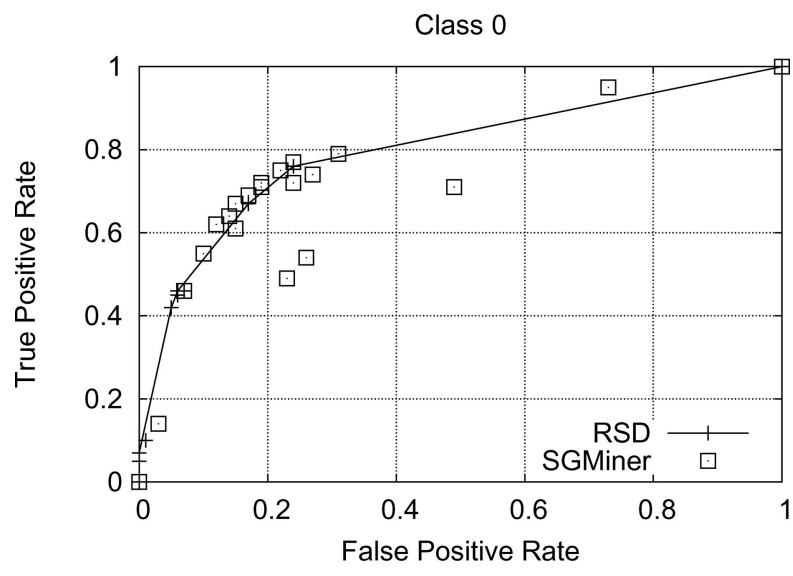

Class 1

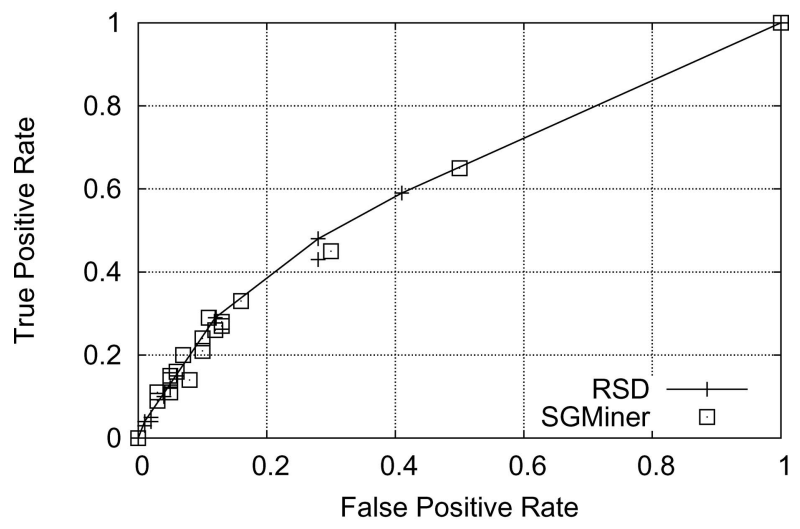

Class 5

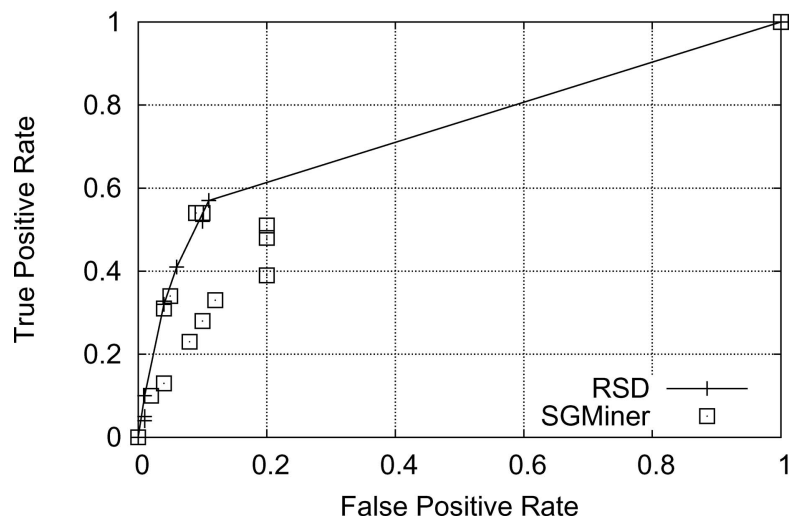

appropriate tradeoff between rule significance, coverage and complexity). It is the default mode in the RSD implementation.

We have successfully applied the RSD algorithm to the Trains, KRK and Mutagenesis benchmark problems. RSD was successfully applied also to the Telecom domain, a real-life data set from a telecommunications company, where the results were evaluated as meanSpringer 
ingful by the domain expert. An experimental comparison with state-of-the-art subgroup discovery system SubgroupMiner was successfully performed in the Traffic problem domain. The results of the experimental comparison with SubgroupMiner indicate that the two systems identify best subgroups on the ROC convex hull of roughly similar quality, but that SubgroupMiner induces a much larger set of subgroups, some lying much below the ROC curve. It is also worth noticing that unlike SubgroupMiner which requires tabular data representation, RSD is readily applicable to Prolog data representations including intensional background predicate definitions.

In further work we will investigate an exhaustive, rather than a heuristic, approach to relational subgroup discovery. Moreover, the idea of incrementally extending the feature set depending on the quality of the discovered subgroups, seems also worth investigating in further work.

Acknowledgments We are grateful to Peter Flach for the collaboration in the genesis phase of the RSD algorithm. The efficient first-order feature construction procedure of RSD is an extension of the feature construction procedure of the $1 \mathrm{BC}$ algorithm by Peter Flach and Nicolas Lachiche. The comparison with SINUS was done in collaboration with Simon Rawles. The constraint-based subgroup discovery framework was elaborated in collaboration with Sašo Džeroski. We are grateful to Jiří Zídek, Atlantis Telecom s.r.o. for the comments on the Telecom subgroups discovered, and to Dietrich Wettschereck for making a procedure for subgroup plotting available for the experiments in the Telecom domain. Nada Lavrač acknowledges the support of the Slovenian Ministry of Higher Education, Science and Technology. Filip Železný was supported by the Czech ministry of education grant 1K04108 Research and Implementation of Efficient Database Propositionalization Methods. The described research was developed in part within the framework of the Slovenian-Czech bilateral project Knowledge Management in Medicine and Health Care (2004-2005).

\section{References}

Agrawal, R., Mannila, H., Srikant, R., Toivonen, H., \& Verkamo, A.I. (1996). Fast discovery of association rules. In Advances in knowledge discovery and data mining (pp. 307-328).

Aronis, J., \& Provost, J. F. (1994). Efficiently constructing relational features from background knowledge for inductive machine learning. In AAAI-94 Workshop on Knowledge Discovery in Databases. (pp. 347-358).

Aronis, J. M., Provost, F. J., \& Buchanan, B. G. (1996). Exploiting background knowledge in automated discovery. In Knowledge discovery and data mining (pp. 355-358).

Bayardo, R. (2002). Editorial: The many roles of constraints in data mining. SIGKDD Explorations, 4(1), i-ii.

Cestnik, B. (1990). Estimating probabilities: A crucial task in machine learning. In Proceedings of the 9th European Conference on Artificial Intelligence (pp. 147-149) Pitman.

Clark, P., \& Boswell, R. (1991). Rule induction with CN2: Some recent improvements. In Proceedings Fifth European Working Session on Learning (pp. 151-163). Berlin, Springer.

Clark, P., \& Niblett, T. (1987). Induction in noisy domains. In Progress in Machine Learning (Proceedings of the 2nd European Working Session on Learning) (pp. 11-30). Sigma Press.

Clark, P., \& Niblett, T. (1989). The CN2 induction algorithm. Machine Learning, 3, 261-283.

Cohen, W. W. (1995). Fast effective rule induction. In A. Prieditis \& S. Russell (Eds.), Proceedings of the 12th International Conference on Machine Learning. Tahoe City, CA (pp. 115-123). Morgan Kaufmann.

Cohen, W. W. \& Singer, Y. (1991). Hypothesis-driven constructive induction in AQ17: A method and experiments. In Proceedings of the IJCAI-91 Workshop on Evaluating and Changing Representations in Machine Learning (pp. 13-22).

De Raedt, L., Blockeel, H., Dehaspe, L., \& Van Laer, W. (2001). Three companions for data mining in first order logic. In: S. Džeroski and N. Lavrač (Eds.), Relational Data Mining (pp. 105-139). Springer-Verlag.

De Raedt, L., \& Dehaspe, L. (1997). Clausal discovery. Machine Learning, 26, 99-146.

Džeroski, S., Cestnik, B., \& Petrovski, I. (1993). Using the m-estimate in rule induction. Journal of Computing and Information Technology, 1:1, 37-46.

Džeroski, S., \& Lavrač N. (Eds.) (2001). Relational Data Mining. Berlin: Springer-Verlag.

Fawcett, T. (2001). Using Rule Sets to Maximize ROC Performance. In Proceedings of the International Conference on Data Mining (pp. 131-138). 
Flach, P., \& Lachiche, N. (1999). 1BC: A First-Order Bayesian Classifier. In S. Džeroski \& P. Flach (Eds.), Proceedings of the 9th International Workshop on Inductive Logic Programming (pp. 92-103). SpringerVerlag.

Flach, P., Mladenić, D. Moyle, Raeymaekers S., Rauch J., Rawles S., Ribeiro R., Sclep G., Struyf J., Todorovski L., Torgo H. B. L., Wettschereck D., Wu S., Gartner T., Grobelnik M., Kavšek B., Kejkula M., Krzywania D., Lavrač N., \& Ljubič P. (2003). On the road to knowledge: Mining 21 years of UK Tra**c Accedents Reports. In: D. Mladenić, N. Lavrač, M. Bohanec, \& S. Moyle (Eds.), Data Mining and Decision Support: Integration and Collaboration (pp.143-156). Kluwer.

Gamberger, D., \& Lavrač, N. (2002). Expert guided subgroup discovery: Methodology and application. Journal of Artificial Intelligence Research, 17, 501-527.

Garofalakis, M., \& Rastogi, R. (2000). Scalable data mining with model constraints. SIKDD Explorations $2: 2,39-48$.

Geibel, P., \& Wysotzki, F. (1996). Learning relational concepts with decision trees. In L. Saitta (Ed.), Proceedings of the 13th International Conference on Machine Learning (pp. 166-174). Morgan Kaufmann.

Imielinsky, T., \& Mannila, H. (1996). A database perspective on knowledge discovery. Communications of the ACM, 39:11, 58-64.

Kavšek, B., \& Lavrač (2004). Analysis of example weighting in subgroup discoveryby comparison of three algorithms on a real-life data set. In J. Fuernkranz (Ed.), Proceedings of the ECML/PKDD Workshop on Advances in Inductive Rule Learning (pp. 64-76).

Kloesgen, W. (1996). EXPLORA: A multipattern and multistrategy discovery assistant. In Advances in Knowledge Discovery and Data Mining. (pp. 249-271). Menlo Park, CA: AAAI Press.

Kloesgen, W., \& May, M. (2002). Census Data Mining-An Application. In Procs. 6th European Conference on Principles and Practice of Knowlede Discovery in Databases.

Koller, D., \& Sahami, M. (1996). Toward optimal feature selection. In Proceedings of the International Conference on Machine Learning (pp. 284-292).

Kramer, S., Lavrač, N., \& Flach, P. (2001). Propositionalization Approaches to Relational Data Mining. In S. Džeroski \& N. Lavrač (Eds.), Relational Data Mining (pp. 262-291). Springer-Verlag.

Kramer, S., Pfahringer, B., \& Helma, C. (1998). Stochastic Propositionalizationof Non-determinate Background Knowledge. In D. Page (Ed.), Proceedings of the 8th International Conference on Inductive Logic Programming, Vol. 1446 of Lecture Notes in Artificial Intelligence (pp. 80-94). Springer-Verlag.

Krogel, M.-A., Rawles, S., \& Železný, F., Flach, P. A., Lavrač, N., \& Wrobel, S. (2003). Comparative evaluation of approaches to propositionalization. In Proceedings of the 13th International Conference on Inductive Logic Programming. Springer-Verlag.

Lavrač, N., \& Džeroski, S. (1994). Inductive Logic Programming: Techniques and Applications. Ellis Horwood.

Lavrač, N. \& Flach, P. A. (2001). An extended transformation approach to inductivelogic programming. ACM Transactions on Computational Logic, 2:4, 458-494.

Lavrač, N., Gamberger, D., \& Jovanoski, V. (1999). A study of relevance for learningin deductive databases. Journal of Logic Programming, 40:2/3, 215-249.

Lavrač, N., Kavšek, B., Flach, P., \& Todorovski, L. (2004). Subgroup Discovery with CN2-SD. Journal of Machine Learning Research, 5, 153-188.

Mannila, H., \& Toivonen, H. (1997). Levelwise search and borders of theories in knowledge discovery. Data Mining and Knowledge Discovery, 1:3, 241-258.

Michie, D., Muggleton, S., Page, D., \& Srinivasan, A. (1994). To the international computing community: A new East-West challenge. Technical report, Oxford University Computing Laboratory, Oxford, UK.

Muggleton, S. (1992). Inductive Logic Programming. Academic Press.

Muggleton, S. (1995). Inverse Entailment and Progol. New Generation Computing, Special issue on Inductive Logic Programming 13:3-4, 245-286.

Muggleton, S., Bain, M., Hayes-Michie, J., \& Michie, D. (1989). An experimentalcomparison of human and machine learning formalism. In Proceedings of the 6th International Workshop on Machine Learning. (pp. 113-118).

Oliveira, A., \& Sangiovanni-Vincentelli, A. (1992). Constructive induction using a non-greedy strategy for feature selection. In Proceedings of the 9th InternationalWorkshop on Machine Learning.

Pagallo, G., \& Haussler, D. (1990). Boolean feature discovery in empirical learning. Machine Learning, 5:1, 71-99.

Provost, F. J., \& Fawcett, T. (1998). Robust classification systems for imprecise environments. In Proceedings of the 15th Conference on Artificial Intelligence (pp. 706-713).

Quinlan, J. (1990). Learning logical definitions from Relations. Machine Learning, 5, 239-266.

Quinlan, J. R. (1993). C4.5: Programs for Machine Learning. Morgan Kaufmann.

Rivest, R. L. (1987). Learning decision lists. Machine Learning 2:3, 229-246. 
Sebag, M., \& Rouveirol, C. (1997). Tractable induction and classification in first-order logic via stochastic matching. In Proceedings of the 15th InternationalJoint Conference on Artificial Intelligence (pp. 888893). Morgan Kaufmann.

Srinivasan, A., \& King, R. (1996). Feature construction with Inductive Logic Programming: A study of quantitative predictions of biological activity aided bystructural attributes. In Proceedings of the 6th International Workshop on Inductive Logic Programming. (pp. 89-104). Springer-Verlag.

Srinivasan, A., Muggleton, S. H., Sternberg, M. J. E., \& King, R. D. (1996). Theories for mutagenicity: A study in first-order and feature-based induction. Artificial Intelligence, 84, 277-299.

Stahl, I. (1996). Predicate invention in inductive logic programming. In L. De Raedt (Ed.), Advances in Inductive Logic Programming. IOS Press (pp. 34-47).

Suzuki, E. (2004). Discovering interesting exception rules with rule pair. In J. Fuernkranz (Ed.), Proceedings of the ECML/PKDD Workshop on Advances in Inductive Rule Learning (pp. 163-178).

Turney, P. (1996). Low size-complexity inductive logic programming: the east-west challenge considered as a problem in cost-sensitive classification. In L. De Raedt (Ed.), Advances in Inductive Logic Programming. IOS Press (pp. 308-321).

Witten, I. H., \& Frank, E. (1999). Data Mining: Practical Machine Learning Toolsand Techniques with Java Implementations. Morgan Kaufmann.

Witten, I. H., Frank, E., Trigg, L., Hall, M., Holmes, G., \& Cunningxham, S. J. (1999). Weka: Practical Machine Learning Tools and Techniques with Java Implementations.

Wrobel, S. (1997). An algorithm for multi-relational discovery of subgroups. In J.Komorowski \& J. Zytkow (Eds.), Proceedings of the First European Symposion on Principles of Data Mining and Knowledge Discovery (PKDD-97) (pp. 78-87). Berlin, Springer Verlag.

Wrobel, S. (2001). Inductive logic programming for knowledge discovery indatabases. In S. Džeroski \& N. Lavrač (Eds.), Relational Data Mining. (pp. 74-101) Springer-Verlag.

Wrobel, S., \& Džeroski, S. (1995). The ILP description learning problem: Towardsa general model-level definition of data mining in ILP. In K. Morik \& J. Herrmann (Eds.), Proceedings of the Fachgruppentreffen Maschinelles Lernen(FGML-95). 44221 Dortmund, Univ. Dortmund.

Železný, F., Mikšovský, P., Štepánková, O., \& Zídek, J. (2000). ILP for automated telephony. In J. Cussens $\&$ A. Frisch (Eds.), Proceedings of the Work-in-Progress Track at the 10th International Conference on Inductive Logic Programming (pp. 276-286).

Železný, F., Zídek, J., \& Štěpánková, O. (2002). A learning system for decision support in telecommunications. In Proceedings of the 1st International Conference on Computing in an Imperfect World, Belfast 4/2002. Springer-Verlag.

Zucker, J.-D., \& Ganascia, J.-G. (1996). Representation changes for efficient learning in structural domains. In L. Saitta (Ed.), Proceedings of the 13th International Conference on Machine Learning (pp. 543-551). Morgan Kaufmann,

Zucker, J.-D., \& Ganascia, J.-G. (1998). Learning structurally indeterminate clauses. In D. Page (Ed.), Proceedings of the 8th International Conference on Inductive Logic Programming (pp. 235-244). SpringerVerlag. 